\title{
Lomustine-temozolomide combination therapy versus standard temozolomide therapy in patients with newly diagnosed glioblastoma with methylated MGMT promoter (CeTeG/NOA-09): a randomised, open-label, phase 3 trial
}

\author{
Herrlinger, Ulrich ; Tzaridis, Theophilos ; Mack, Frederic ; et al ; Weller, M
}

\begin{abstract}
BACKGROUND There is an urgent need for more effective therapies for glioblastoma. Data from a previous unrandomised phase 2 trial suggested that lomustine-temozolomide plus radiotherapy might be superior to temozolomide chemoradiotherapy in newly diagnosed glioblastoma with methylation of the MGMT promoter. In the CeTeG/NOA-09 trial, we aimed to further investigate the effect of lomustine-temozolomide therapy in the setting of a randomised phase 3 trial. METHODS In this open-label, randomised, phase 3 trial, we enrolled patients from 17 German university hospitals who were aged 18-70 years, with newly diagnosed glioblastoma with methylated MGMT promoter, and a Karnofsky Performance Score of $70 \%$ and higher. Patients were randomly assigned (1:1) with a predefined SAS-generated randomisation list to standard temozolomide chemoradiotherapy $(75 \mathrm{mg} / \mathrm{m} \mathrm{per}$ day concomitant to radiotherapy [59-60 Gy] followed by six courses of temozolomide 150-200 mg/m per day on the first 5 days of the 4 -week course) or to up to six courses of lomustine $(100 \mathrm{mg} / \mathrm{m}$ on day 1$)$ plus temozolomide (100-200 mg/m per day on days 2-6 of the 6 -week course) in addition to radiotherapy (59-60 Gy). Because of the different schedules, patients and physicians were not masked to treatment groups. The primary endpoint was overall survival in the modified intention-to-treat population, comprising all randomly assigned patients who started their allocated chemotherapy. The prespecified test for overall survival differences was a log-rank test stratified for centre and recursive partitioning analysis class. The trial is registered with ClinicalTrials.gov, number NCT01149109. FINDINGS Between June 17, 2011, and April 8, 2014, 141 patients were randomly assigned to the treatment groups; 129 patients (63 in the temozolomide and 66 in the lomustine-temozolomide group) constituted the modified intention-to-treat population. Median overall survival was improved from $31 \cdot 4$ months (95\% CI $27 \cdot 7$ $47 \cdot 1)$ with temozolomide to $48 \cdot 1$ months $(32 \cdot 6$ months-not assessable) with lomustine-temozolomide (hazard ratio $[\mathrm{HR}] 0 \cdot 60,95 \%$ CI $0 \cdot 35-1 \cdot 03 ; \mathrm{p}=0 \cdot 0492$ for log-rank analysis). A significant overall survival difference between groups was also found in a secondary analysis of the intention-to-treat population $(\mathrm{n}=141$, HR $0 \cdot 60,95 \%$ CI $0 \cdot 35-1 \cdot 03 ; \mathrm{p}=0 \cdot 0432$ for log-rank analysis). Adverse events of grade 3 or higher were observed in $32(51 \%)$ of 63 patients in the temozolomide group and $39(59 \%)$ of 66 patients in the lomustine-temozolomide group. There were no treatment-related deaths. INTERPRETATION Our results suggest that lomustine-temozolomide chemotherapy might improve survival compared with temozolomide standard therapy in patients with newly diagnosed glioblastoma with methylated MGMT promoter. The findings should be interpreted with caution, owing to the small size of the trial. FUNDING German Federal Ministry of Education and Research.
\end{abstract}

DOI: https://doi.org/10.1016/S0140-6736(18)31791-4 
Journal Article Accepted Version

\section{(c) $\mathbb{Q} \otimes \Theta$}

The following work is licensed under a Creative Commons: Attribution-NonCommercial-NoDerivatives 4.0 International (CC BY-NC-ND 4.0) License.

Originally published at:

Herrlinger, Ulrich; Tzaridis, Theophilos; Mack, Frederic; et al; Weller, M (2019). Lomustine-temozolomide combination therapy versus standard temozolomide therapy in patients with newly diagnosed glioblastoma with methylated MGMT promoter (CeTeG/NOA-09): a randomised, open-label, phase 3 trial. The Lancet, 393(10172):678-688.

DOI: https://doi.org/10.1016/S0140-6736(18)31791-4 


\section{Phase III trial of CCNU/temozolomide (TMZ) combination therapy vs. standard TMZ therapy for newly diagnosed MGMT-methylated glioblastoma patients: the randomized, open-label CeTeG/NOA-09 trial}

Ulrich Herrlinger, Prof. M.D. ${ }^{1 *}$ Theophilos Tzaridis, M.D., ${ }^{1 *}$ Frederic Mack, M.D., ${ }^{1}$ Joachim Peter Steinbach, Prof. M.D., ${ }^{7}$ Uwe Schlegel, Prof. M.D., ${ }^{8}$ Michael Sabel, M.D., ${ }^{9}$ Peter Hau, Prof. M.D., ${ }^{10}$ Rolf-Dieter Kortmann, Prof. M.D., ${ }^{11}$ Dietmar Krex, M.D., ${ }^{12}$ Oliver Grauer, M.D. Ph.D., ${ }^{13}$ Roland Goldbrunner, Prof. M.D., ${ }^{15}$ Oliver Schnell, M.D., ${ }^{17,22}$ Oliver Bähr, M.D., ${ }^{7}$ Martin Uhl, M.D., ${ }^{10}$ Clemens Seidel, M.D., ${ }^{11}$ Ghazaleh Tabatabai, Prof. M.D. Ph.D., ${ }^{18}$ Thomas Kowalski, M.D., ${ }^{8}$ Florian Ringel, Prof. M.D., ${ }^{19,23}$ Friederike Schmidt-Graf, M.D., ${ }^{20}$ Bogdana Suchorska, M.D., ${ }^{17}$ Stefanie Brehmer, M.D., ${ }^{21}$ Astrid Weyerbrock, M.D., ${ }^{22}$ Miriam Renovanz, M.D. ${ }^{23}$ Lars Bullinger, Prof. M.D. ${ }^{24}$ Norbert Galldiks, M.D., ${ }^{16,30}$ Peter Vajkoczy, Prof. M.D., ${ }^{25}$ Martin Misch, M.D., ${ }^{25}$ Hartmut Vatter, Prof. M.D., ${ }^{2}$ Moritz Stuplich, M.D., ${ }^{1}$ Niklas Schäfer, M.D.,${ }^{1}$ Sied Kebir, M.D., ${ }^{1}$ Johannes Weller, M.D., ${ }^{1}$ Christina Schaub, M.D., ${ }^{1}$ Walter Stummer, Prof. M.D. ${ }^{14}$ Jörg-Christian Tonn, Prof. M.D., ${ }^{17}$ Matthias Simon, M.D.. ${ }^{2 \# ~ V e r a ~}$ C. Keil, M.D., ${ }^{3}$ Michael Nelles, M.D., ${ }^{3}$ Horst Urbach, Prof. M.D., ${ }^{3,31}$ Martin Coenen, M.D., ${ }^{6}$ Wolfgang Wick, Prof. M.D., ${ }^{26}$ Michael Weller, Prof. M.D., ${ }^{27}$ Rolf Fimmers, Ph.D., ${ }^{4}$ Matthias Schmid, Prof. Ph.D., ${ }^{4}$ Elke Hattingen, Prof. M.D., ${ }^{3}$ Torsten Pietsch, Prof. M.D., ${ }^{5}$ Christoph Coch, M.D., ${ }^{6 *}$ Martin Glas, M.D. ${ }^{1,28^{*}}$ for the Neurooncology Working Group (NOA) of the German Cancer Society

\footnotetext{
${ }^{1}$ Division of Clinical Neurooncology, Department of Neurology and Center of Integrated Oncology (CIO), Departments of ${ }^{2}$ Neurosurgery and ${ }^{3}$ Neuroradiology, ${ }^{4}$ Institute for Medical Biometry, Informatics and Epidemiology, ${ }^{5}$ Institute of Neuropathology and DGNN Brain Tumor Reference Center, ${ }^{6}$ Study Center Bonn, University Hospital Bonn, Sigmund-Freud Str. 25, 53127 Bonn, Germany

${ }^{7}$ Dr. Senckenberg Institute of Neurooncology, University of Frankfurt, Schleusenweg 2-16, 60528 Frankfurt am Main, Germany, ${ }^{8}$ Department of Neurology, University Hospital Knappschaftskrankenhaus, Ruhr-Universität Bochum, In der Schornau 23-25, 44892 Bochum, Germany

${ }^{9}$ Department of Neurosurgery, University of Düsseldorf, Moorenstr. 5, 40255 Düsseldorf, Germany

${ }^{10}$ Department of Neurology and Wilhelm Sander NeuroOncology Unit, University Hospital Regensburg, Universitätsstraße 84, 93053 Regensburg, Germany

${ }^{11}$ Department of Radiation Oncology, University of Leipzig, Stephanstraße 9, 04103 Leipzig, Germany

${ }^{12}$ Department of Neurosurgery, University of Dresden, Fetscherstr. 74, 01307 Dresden, Germany

Departments of ${ }^{13}$ Neurology and ${ }^{14}$ Neurosurgery, University of Münster, Albert-Schweitzer-Campus 1, 48149 Münster, Germany

Departments of ${ }^{15}$ Neurosurgery and ${ }^{16}$ Neurology, University of Cologne, Kerpener Str. 62, 50937 Cologne, Germany

${ }^{17}$ Department of Neurosurgery, Ludwig Maximillian University of Munich and German Cancer Consortium (DKTK), Partner Site Munich, Marchioninistr. 15, 81377 Munich, Germany

${ }^{18}$ Interdisciplinary Division of Neurooncology, University of Tübingen, Hoppe-Seyler-Str. 3, 72076 Tübingen, Germany

Departments of ${ }^{19}$ Neurosurgery and ${ }^{20}$ Neurology, Technical University of Munich, Ismaningerstr. 22, 81675 Munich, Germany

${ }^{21}$ Department of Neurosurgery, University of Mannheim, Theodor-Kutzer-Ufer 1-3, 68167 Mannheim, Germany

Departments of ${ }^{22}$ Neurosurgery and ${ }^{31}$ Neuroradiology, University of Freiburg, Breisacher Str. 64, 79106 Freiburg, Germany

${ }^{23}$ Department of Neurosurgery, University of Mainz, Langenbeckstr. 1, 55101 Mainz, Germany

${ }^{24}$ Department of Internal Medicine, University of Ulm, Albert-Einstein Allee 23, $89081 \mathrm{Ulm}$, Germany

${ }^{25}$ Department of Neurosurgery, Charité University of Berlin, Charitéplatz 1, 10117 Berlin, Germany

${ }^{26}$ Department of Neurology, University of Heidelberg and German Cancer Research Center (DKFZ), Im Neuenheimer Feld 400, 69120 Heidelberg, Germany

${ }^{27}$ Department of Neurology, University Hospital and University of Zurich, Frauenklinikstr. 26, 8091 Zurich, Switzerland

${ }^{28}$ Division of Clinical Neurooncology, Department of Neurology and ${ }^{29}$ West German Cancer Center (WTZ), German Cancer Consortium, Partner Site Essen, University Hospital Essen, University Duisburg-Essen, Hufelandstr. 55, 45147 Essen, Germany ${ }^{30}$ Institute of Neuroscience and Medicine (INM-3), Forschungszentrum Juelich, Wilhelm-Johnen-Str., 52425 Juelich, Germany *contributed equally, "present address: Department of Neurosurgery, Evangelisches Klinikum Bethel, Burgsteig 13, 33617 Bielefeld, Germany
}

Corresponding author:

Ulrich Herrlinger, M.D.

Professor of Clinical Neurooncology

Division of Clinical Neurooncology

Department of Neurology and Center of Integrated Oncology

University of Bonn Medical Center

Sigmund-Freud-Str. 25

D-53105 Bonn, Germany

Tel.: +49-228-2873 1241

Fax: +49-228-2871 9043

e-mail: ulrich.herrlinger@ukbonn.de 


\section{Abstract}

BACKGROUND: There is an urgent need for more effective therapies in glioblastoma. Data from a previous unrandomized phase II trial (UKT-03) suggested that lomustine/temozolomide (CCNU/TMZ) plus radiotherapy may be superior to temozolomide (TMZ) chemoradiotherapy in newly diagnosed O6-methylguanine DNA-methyltransferase (MGMT) promotor-methylated glioblastoma. The aim of the CeTeG/NOA-09 trial was to further investigate CCNU/TMZ in a confirmatory randomized phase III trial.

METHODS: The open-label CeTeG/NOA-09 phase III trial randomized (1:1) patients with newly diagnosed MGMT promotor-methylated glioblastoma (age 18-70, Karnofsky Performance Score $70 \%$ and higher) to standard TMZ chemoradiotherapy (TMZ therapy $75 \mathrm{mg} / \mathrm{m}^{2} / \mathrm{d}$ concomitant to radiotherapy $59 \cdot 4-60$ Gy, followed by 6 courses TMZ $150-200 \mathrm{mg} / \mathrm{m}^{2} / \mathrm{d}$ days $\left.1-5 / 28\right)$ or to up to six courses of CCNU (100 $\mathrm{mg} / \mathrm{m}^{2}$ day 1$)$ plus TMZ (100 mg/m² days $\left.2-6 / 42\right)$ in addition to radiotherapy (59.460 Gy). The primary endpoint was overall survival (OS) in the modified intention-totreat $(\mathrm{mlTT})$ population comprising all randomized patients having started with their allocated chemotherapy. The prespecified test for OS differences was a log-rank test stratified for center and recursive partitioning analysis group. The trial is registered with ClinicalTrials.gov NCT01149109.

FINDINGS: The trial randomized 141 patients; 129 patients (63 TMZ, 66 CCNU/TMZ) constituted the mITT population. Overall survival was superior in the CCNU/TMZ arm $(p=0 \cdot 0492)$. Median OS was prolonged from 31.4 months $(95 \% \mathrm{Cl} 27 \cdot 7-47 \cdot 1$ months) with TMZ to 48.1 months (95\% Cl 32.6 months-not assessable) with 
CCNU/TMZ. The hazard ratio for death was $0.60(95 \% \mathrm{Cl} 0.35-1.03)$ in the CCNU/TMZ arm as compared to the TMZ arm. A significant OS difference between arms was also found in a secondary analysis of the as-randomized population $(n=141 ; p=0.0432 ; H R 0.60,95 \% \mathrm{Cl} 0.35-1 \cdot 03)$. Adverse events grade 3 or higher were observed in $50.8 \%$ of patients with TMZ and $59.1 \%$ of patients with CCNU/TMZ.

INTERPRETATION: CCNU/TMZ chemotherapy may improve efficacy as compared to TMZ standard therapy in patients with newly diagnosed MGMT promotormethylated glioblastoma.

FUNDING: German Federal Ministry of Education and Research 


\section{Research in context}

\section{Evidence before this study}

Prior to the trial, the standard therapy for newly diagnosed glioblastoma patients was radiotherapy (59.4-60 Gy) with concomitant daily low-dose $\left(75 \mathrm{mg} / \mathrm{m}^{2} / \mathrm{d}\right)$ temozolomide chemotherapy, followed by 6 courses of adjuvant temozolomide therapy (150-200 mg/m²/d d1-5, 28-day course). The MGMT promotor methylation status is a predictor for the benefit from temozolomid therapy: MGMT promotormethylated patients have a particularly high survival benefit from TMZ therapy. A single-arm phase II trial (UKT-03) applying CCNU/temozolomide combination therapy to newly diagnosed glioblastoma patients found a signal of prolonged overall survival for patients with MGMT promotor-methylated glioblastoma. Based on this, the CeTeG/NOA-09 trial further analyzed the value of the CCNU/temozolomide combination chemotherapy in MGMT promotor-methylated glioblastoma patients in a randomized phase III setting.

\section{Added value of this study}

The predefined final analysis of the primary endpoint confirmed that combined CCNU/temozolomide chemotherapy plus radiotherapy may improve overall survival as compared to standard temozolomide chemotherapy plus radiotherapy.

\section{Implications of all the available evidence}

CCNU/temozolomide combination chemotherapy prolonged survival in a selected group of MGMT promoter-methylated patients in this small randomized trial. These encouraging results require further confirmation; if confirmed, this regimen has the potential to become a standard of care option. Ongoing research aims at further 
investigating the molecular determinants of response to $\mathrm{CCNU} /$ temozolomide and the cellular changes induced by combined CCNU/temozolomide chemotherapy. 


\section{Introduction}

Chemotherapy for newly diagnosed glioblastoma has not been substantially improved since the registration trial for temozolomide (TMZ). ${ }^{1}$ The addition of tumortreating fields to TMZ was associated with moderate survival prolongation. ${ }^{2}$ Randomized trials using dose-intensified TMZ regimens ${ }^{3}$ or combining TMZ with other drugs ${ }^{4-7}$ have all failed to prolong overall survival (OS). Nevertheless, the comparably low toxicity of TMZ suggests that more intense alkylating combination therapy may be feasible and should be further investigated.

Nitrosourea compounds are well established in glioma therapy. ${ }^{8-12}$ They are capable to penetrate the brain via an intact blood-brain-barrier. The combination of nitrosoureas with TMZ is not a mere dose escalation of alkylating therapy but may also combine different qualities of DNA damage with the potential for additive or even synergistic effects. In contrast to TMZ exerting its therapeutic effect preferably through alkylation of guanine, lomustine (CCNU) has effects beyond DNA alkylation: It acts as an bifunctional agent introducing interstrand crosslinks ${ }^{13}$ and leads to carbamoylation of amino acids thus interfering with transcriptional, translational and posttransscriptional processes. ${ }^{14}$ In contrast to the alkylating mode of action shared by TMZ and nitrosureas, the non-alkyating modes of action may not depend on the O6-methylguanine DNA-methyltransferase (MGMT) promotor methylation status and the MGMT enzyme activity which counteracts guanine alkylation. It is therefore not surprising that combined nitrosourea and TMZ therapy showed enhanced activity in high-grade glioma xenograft models. ${ }^{15}$ Also, a single-arm trial with BCNU and unescalated TMZ provided promising results in patients with inoperable 
gllioblastoma. ${ }^{16}$ These concepts and experimental results provide a clear rationale to evaluate the efficacy of combined CCNU/TMZ therapy in glioblastoma patients.

The single-arm phase II UKT-03 trial ${ }^{17,18}$ included 31 patients and explored the value of a combined CCNU/TMZ chemotherapy in newly diagnosed glioblastoma. In line with previous trials using nitrosoureas ${ }^{8,9}$ which defined the standard in 2002 when UKT-03 started, chemotherapy started with the first course during RT. WIth CCNU/TMZ combination therapy, UKT-03 found a signal for prolonged OS with a median of 23 months as opposed to 15-17 months in contemporary historical controls. Yet, OS prolongation was exclusively seen in patients with MGMT promotor-methylated glioblastoma. Their median OS was 34.5 months comparing favorably to 23.4 months in the TMZ registration trial ${ }^{19}$ while median OS remained at 12.5 months in MGMT promotor-unmethylated glioblastoma. ${ }^{18} \mathrm{~A}$ favorable OS signal was also seen in a non-randomized trial applying CCNU/TMZ to pediatric high-grade glioma patients. ${ }^{20}$ The encouraging data of UKT-03 led to the CeTeG/NOA-09 trial which tests whether CCNU/TMZ is superior to TMZ standard therapy in a randomized phase III setting. CeTeG/NOA-09 implemented exactly the CCNU/TMZ treatment regime of the UKT-03 trial (including omission of RT-concomitant daily chemotherapy) and was restricted to patients with MGMT promotor-methylated glioblastoma on the base of the previous UKT-03 subgroup analyses. 


\section{Patients and methods}

\section{Trial oversight}

CeTeG/NOA-09 is a randomized phase III trial (EudraCT No. 2009-011252-22; ClinicalTrials.gov NCT01149109, protocol available at https://neurologie.unibonn.de/sektionen/klinische-neuroonkologie/therapiestudien.htm) approved by Ethic Committees of all 17 participating centers. All patients gave written informed consent. All trial procedures adhered to the Declaration of Helsinki and the Guidelines for Good Clinical Practice. An independent Data Monitoring and Safety Board reviewed all safety-relevant information every 6 months.

\section{Sample size calculation}

CeTeG/NOA-09 had to recruit 128 evaluable patients. The sample size calculation was performed using the "PS" power and sample size program. ${ }^{21}$ The sample size was based on the assumption that CCNU/TMZ could increase 2-year OS from $48.9 \%{ }^{18}$ to $70 \%$ (UKT-03: $\left.75 \%\right) .{ }^{18}$ Assuming exponentially distributed survival times (event/death rates 0.356 and 0.176 per patient year, hazard ratio 0.50 ), a constant recruitment of 64 evaluable patients per treatment group (+ 4 early dropouts per group) over two years with a follow-up of at least two years results in a power of $80 \%$ for the intended two-sided log-rank test (significance level 0.05). The recruitment period initially planned for 24 months (04/2011-04/2013) had to be prolonged until 06/2014 (last-patient-in) since the rate of MGMT promotor-methylated tumors $(35.8 \%)$ was lower than previously reported $(45 \%) .{ }^{19}$ The follow-up time of initially planned 24 months after last-patient-in had to be prolonged to 34 months $(04 / 2017$ closure of the trial as planned), since a treatment arm-blinded analysis of OS 14 
months after last-patient-in showed a low overall mean risk for death of $0 \cdot 1994 /$ patient year. The prolongation of the follow-up time to 34 months allowed to retain the power of $80 \%$ despite the lower event rates.

\section{Participants}

Patients were recruited in 17 German University Hospitals based on the following inclusion criteria: no prior chemotherapy or radiotherapy (RT), age 18-70 years, newly diagnosed glioblastoma or gliosarcoma (resection or biopsy) centrally confirmed (TP, for details see Web Appendix), methylated MGMT promotor according to central testing (MDXHealth, Herstal, Belgium, for details see below); Karnofsky performance score $(\mathrm{KPS}) \geq 70 \%$; stable or decreasing corticosteroids within 5 days prior to randomization; adequate hematologic, hepatic, renal and coagulation function. Exclusion criteria included prior malignancy treated less than 5 years ago, prior medical treatment for any cancer, severe other psychological, cognitive, familial, sociological or geographical condition potentially interfering with compliance with the study protocol, any other antitumor therapy not described in the protocol.

\section{Molecular tumor assessment}

Prior to randomization, tumor specimens were analyzed centrally for MGMT promotor methylation using real-time methylation-specific $\mathrm{PCR}^{21}$ and tumors were classified as MGMT promotor-methylated if the ratio of MGMT to the $\beta$-actin reference gene $(A C T B)$ was $>2$ calculated as (methylated MGMT/ACTB) $\times 1000 .{ }^{19,22,23}$ Responding to changes implemented by the WHO classification of brain tumors $2016,{ }^{24}$ all available tumor tissue of patients in the trial was retrospectively reclassified. Analyses for $\alpha$-thalassemia/mental-retardation-syndrome-X-linked (ATRX) loss 
(immunohisto-chemistry, MAb clone CL0537, Sigma, St. Louis, U.S.A.) and isocitrate dehydrogenase (IDH) mutation were performed. IDH mutation analysis was started with immunohistochemistry (R132H-specific antibody). ${ }^{25} \mathrm{R} 132 \mathrm{H}$-immunonegative tumors of patients $<55$ years underwent $I D H 1 / 2$ pyrosequencing and, in $I D H$ mutated cases, $1 p / 19 q$ codeletion analysis by the multiplex ligation-dependent probe amplification method (SALSA probe mix P088; MRC Holland, Amsterdam, The Netherlands).

\section{Randomization and treatment}

The CeTeG/NOA-09 trial is an open-label trial. Patients were randomized (1:1) according to a predefined SAS-generated randomization list (fax response from the Study Center Bonn, for details see Web Appendix). Involved-field RT (59.4-60 Gy in 30-33 single day fractions) started 22-35 days after surgery. In the standard TMZ arm, patients additionally had daily concomitant TMZ $\left(75 \mathrm{mg} / \mathrm{m}^{2}\right)$ followed by 6 courses of TMZ (150-200 mg/m²/d for 5 days q4w)..$^{1}$ In the experimental CCNU/TMZ arm, patients received up to 6 six-week courses of CCNU/TMZ (CCNU $100 \mathrm{mg} / \mathrm{m}^{2}$ $\mathrm{d} 1$, TMZ $\left.100 \mathrm{mg} / \mathrm{m}^{2} \mathrm{~d} 2-6\right)$. The first course started on the first day of RT, no daily concomitant TMZ therapy was applied (Fig. 2). In case the nadir (white blood count $(\mathrm{WBC})<1500 / \mu \mathrm{l}$ or thrombocytes $<50000 / \mu \mathrm{l})$ occurred after day 25 , CCNU was reduced by one dose level with the dose levels being $100 \%, 75 \%$ and $50 \%$ of the initial dose. In case of WBC $<1500 / \mu$ l or thrombocytes $<50000 / \mu$ lat the dose level of $50 \%$, CCNU had to be permanently discontinued. Depending on the nadirs during the first 25 days of the previous course, TMZ had to be decreased to the lower dose levels of $75 \mathrm{mg} / \mathrm{m}^{2}$ or $50 \mathrm{mg} / \mathrm{m}^{2}$ or increased stepwise to the higher dose levels of $120 \mathrm{mg} / \mathrm{m}^{2}, 150 \mathrm{mg} / \mathrm{m}^{2}$ and $200 \mathrm{mg} / \mathrm{m}^{2}$ according to the following schedule: reduction by 1 dose level if WBC was $<1500 / \mu$ l or platelets $<50000 / \mu l$; reduction by 2 dose 
levels if WBC was $<1000 / \mu$ l or platelets $<25000 / \mu l$; increase by 1 dose level if RT was completed and WBC was $>2500 / \mu$ l and platelets $>100000 / \mu$ l. In case of WBC $<1500 / \mu$ or thrombocytes $<50000 / \mu \mathrm{l}$ at the lowest dose level of $50 \mathrm{mg} / \mathrm{m}^{2}, \mathrm{TMZ}$ had to be permanently discontinued. In case of any non-hematological toxicity CTCAE grade $3 / 4$, the substance causing the toxicity had to be withheld in further courses and therapy within the trial may continue with the substance not causing the toxicity. The choice of postprogression therapy was left to the treating physician and had to be documented at all visits.

\section{Assessments}

Patients were followed by neurological examination and KPS at baseline, at the beginning of each course and every 12 weeks after completion of chemotherapy. Contrast-enhanced magnetic resonance imaging (MRI) was performed every 12 weeks until death. Determination of progression (PD) was based on RANO criteria ${ }^{26}$ with the following modifications: (1) Up to 12 weeks after completion of RT, PD was only considered for new enhancing lesions outside the radiation field (i.e. beyond the $80 \%$ isodose) or unequivocal histological demonstration of viable tumor. According to previous experience with late pseudoprogression, ${ }^{27}$ PD between week 12 and 24 after completion of RT could only be diagnosed if confirmed 4 to 6 weeks later by another MRI showing further PD. Progressive disease (PD) had to be confirmed by central reference neuroradiology blinded to the protocol $(H U, E H)$. Adverse events (AE; CTCAE criteria version 4.0) were recorded until at least 30 days after the end of study therapy. 


\section{Primary and secondary endpoint analyses}

All statistical analyses were performed by an experienced statistician who is one of the coauthors (RF). The primary endpoint was OS as measured from day of randomization to death or last observation. The modified intent-to-treat population (mITT) including all randomized patients who received their first dose of study chemotherapy (Fig. 1, CONSORT diagram) represented the population on which, according to the protocol, the confirmatory analysis had to be performed. Secondary analyses were performed on the as-randomized population (i.e. population usually named as the ITT population) and the subpopulation of patients with isocitrate dehydrogenase (IDH)1/2 wildtype (wt) tumors. The pre-specified confirmatory analysis was performed using a log-rank test with stratification by center and recursive partitioning analysis (RPA) group. ${ }^{28}$ RPA III implies age $<50$ years + KPS 90-100\%, RPA IV age $<50$ years + KPS $70-80 \%$ or age $>50$ years + at least partial resection + Mini Mental State Examination (MMSE) 27+ points, RPA V age $>50$ years + MMSE $<27$ or age $>50$ and biopsy only. All centers with less than 3 randomized patients per arm were taken together as one center so that the log-rank analysis had 11 categories for the feature "center". Prespecified OS analyses in the mITT population also included Cox regression analyses yielding estimated hazard ratios (HR) with $95 \%$ confidence intervals. Survival was plotted according to the Kaplan Meier method. In line with the log-rank test stratified for center and RPA class, the graphs included only those patients with control counterparts in the respective center $x$ RPA class strata thus enabling a balanced analysis and visualization of survival. Progression-free survival (PFS) was a secondary endpoint and analyzed using the same methods as described above. Secondary endpoints included best response rate as determined by modified RANO criteria in patients with incomplete tumor resection and documented postoperative residual disease, frequency of delay of the 
next chemotherapy course by more than 2 weeks, and acute toxicity according to CTCAE V4.0. Further exploratory analyses included pseudoprogression rates and application of postprogression therapy. Quality of life (QoL) as determined by EORTC QLQ C30 and BN20 questionnaires evaluation of neurotoxicity by neurocognitive testing using the Mini Mental State Examination and a neurcognitive test battery including Trial Making Test A and B, digit span forward and backwards, Controlled Word Association Test for semantic word fluency (animals, food) and lexical word fluency were additional secondary endpoints.

\section{Role of the funding source}

The German Federal Ministry of Education and Research is a non-commercial funder and had no role in study design, collection, analysis and interpretation of data, writing of the manuscript, and the decision to submit the manuscript for publication. The corresponding author $(\mathrm{UH})$ had the final responsibility for all these steps including submission of the manuscript. After data bank closure, UH, TT, RF, CC, MG had access to all trial data. 


\section{Results}

\section{Patients' characteristics}

Between 04/2011 and 04/2014, 657 patients in 17 study centers gave informed consent for the processing of their tumor tissue in the CeTeG trial. In 4 cases, obligatory central reference neuropathology did not confirm glioblastoma histology (3 anaplastic astrocytoma, 1 pilocytic astrocytoma) and these patients were not considered for any further steps in the trial such as MGMT analysis or randomization. Thus, 653 patients with confirmed glioblastoma entered central MGMT promotor methylation analysis (Fig. 1, CONSORT diagram) and 141 patients with MGMT promotor-methylated glioblastoma were randomized. The mITT population comprised 129 patients (63 TMZ, $66 \mathrm{CCNU} / \mathrm{TMZ}$ ). Table 1 shows that the trial predominantly included patients with a high performance score (KPS 90-100\% in $82 \%)$ and a high rate of complete resections (61\%). The distribution of sex was imbalanced between the arms but not relevant for OS in the mITT cohort (HR 0.99, $95 \% 0.63-1.57, \mathrm{p}=0.98$ ) and in the treatment arms (data not shown). There was an imbalance of RPA class distribution in 3 large centers (40/129 patients): here, all 17 patients with RPA 3 or 5 were randomized for CCNU/TMZ while the TMZ standard arm only comprised RPA4 patients. In accordance with the 2007 WHO classification of CNS tumors ${ }^{29}$ applicable throughout the recruiting and treatment phase, CeTeG included 6 patients having a glioblastoma with oligodendroglial component (GBM-O). All 6 GBM-O were IDH mutant and 1p/19q codeleted and thus retrospectively reclassified as anaplastic oligodendroglioma (Table 1). 


\section{Treatment}

Fifty-nine of 63 patients in the standard arm (93.7\%) and 60 of 66 patients in the experimental arm (90.9\%) completed radiotherapy as required with a total dose of 59.4-60 Gy. Sixty percent of patients with TMZ and 39\% with CCNU/TMZ had all 6 chemotherapy courses. The median number of courses was 6 (TMZ) and 5 (CCNU/TMZ). The maximum dose level of $200 \mathrm{mg} / \mathrm{m}^{2} \mathrm{TMZ}$ was achieved in $66.7 \%$ (TMZ) and $37.9 \%$ (CCNU/TMZ) of patients. Dose reductions below $100 \mathrm{mg} / \mathrm{m}^{2} / \mathrm{d}$ occurred only with CCNU/TMZ (TMZ in $12 \cdot 2 \%$, CCNU in $26 \%$ of patients). Further details on dose adjustments (Suppl. Table 1) and mean cumulative daily chemotherapy doses (Suppl. Table 2) are provided in the Appendix. The median length of courses was 28 days (range 26-111 days) with TMZ and 42 days (range 36-84 days) with CCNU/TMZ. During courses 4 to 6 , the percentage of patients with courses substantially delayed for 2-6 weeks was higher with CCNU/TMZ (e.g. 40\% in course 5) than with TMZ (17\% course 5; Suppl. Table 4).

\section{Overall survival}

In the pre-specified confirmatory analysis (log-rank test stratified for center and RPA class), OS was significantly prolonged with CCNU/TMZ as compared to TMZ $(p=0.0492)$. A Kaplan-Meier OS graph including all patients of the mITT population with control counterparts in the respective center $x$ RPA class strata is shown in Fig. 3a. Median OS as taken from this graph is prolonged from 31.4 months $(95 \% \mathrm{Cl}$ $27 \cdot 7-47 \cdot 1$ months) with TMZ to $48 \cdot 1$ months (95\% Cl $32 \cdot 6$ months-not assessable) with CCNU/TMZ. A multivariable Cox regression analysis with center and RPA class as covariates in the mITT population yielded a HR of $0.60(95 \% \mathrm{Cl} 0.35-1.03$; 
$p=0.0641$ ). A significant OS difference between treatment arms was also found in the as-randomized population ( $=$ ITT population, $n=141 ; p=0.0432$, stratified logrank test, fig. 3b), Median OS as taken from fig. $3 \mathrm{~b}$ is prolonged from 30.4 months $(95 \% \mathrm{Cl}$ 27.0-44.9 months) with TMZ to 46.9 months (95\% Cl 31.0-not assessable) with CCNU/TMZ. In this population, the HR was $0.60(95 \% \mathrm{Cl} 0 \cdot 35-1 \cdot 03)$.

In post-hoc sensitivity analyses, a univariate Cox regression analysis for OS in the mITT population revealed a HR of $0.90(95 \% \mathrm{Cl} 0.58-1.41)$. Supplementary Fig. 1a shows the Kaplan Meier OS graph corresponding to an unstratified OS analysis of the mITT population. In this graph, median OS is 31.4 months with TMZ $(95 \% \mathrm{Cl}$ 27.0-44.8 months) and 37.9 months with CCNU/TMZ (95\% Cl 29.2-51.4 months, $\mathrm{p}=0.6579$ ). Additional exploratory post-hoc OS graphs taking into account the imbalance of RPA class distribution in some centers showed a separation of OS curves in favour of the CCNU/TMZ arm: a Kaplan Meier graph with inverse probability weights and inclusion of all 129 mITT patients into the analysis (Suppl. Fig 1b), and a Kaplan Meier graph (89 patients) excluding the 3 centers where the standard arm contains only RPA4 patients but no RPA3 or RPA5 patients (Suppl. Fig. 1c).

In the mITT subpopulation of patients with IDH-wt glioblastoma $(\mathrm{n}=103)$ overall survival was prolonged $(p=0.0374$, stratified log-rank test; HR $0.57,95 \% \mathrm{Cl} 0.30$ $1 \cdot 05$, post-hoc analysis). Thus, the inclusion of 6 patients with GBM-O, nowadays reclassified as anaplastic oligodendroglioma, and 8 patients with IDH-mut glioblastoma, had no influence on the primary endpoint OS. 


\section{Best response and progression-free survival}

Best respone according to RANO criteria was evaluated in the 50 patients of the mITT population (23 TMZ; $27 \mathrm{CCNU/TMZ)} \mathrm{who} \mathrm{were} \mathrm{randomized} \mathrm{with} \mathrm{a} \mathrm{less} \mathrm{than}$ complete resection (table 1). Three patients (13\%) had a partial response upon standard TMZ; four patients (14.8\%) having CCNU/TMZ had at least a partial response, three of them (11.1\%) a complete response. Progression-free survival in the mITT population ( $p=0.4113$, stratified logrank test, fig. $3 c)$ and in the asrandomized population (=ITT population; $p=0.4735$, fig. $3 c$ ) did not differ between the treatment arms. In the mITT population, median PFS was 16.7 months $(95 \% \mathrm{Cl}$ 11.4-24.2 months) with TMZ and 16.7 months (95\% Cl 12-32 months) with CCNU/TMZ. The HR was 0.91 (95\% $\mathrm{Cl} 0.57-1.44$; mITT population) and 0.99 (95\% Cl 0.68-1.46; as-randomized/ITT population). Pseudoprogression confirmed by central reference neuroradiology (according to RANO) or histology (predominance of therapy-induced changes) was found in 5 patients (7.9\%) with TMZ and 7 (10.6\%) with CCNU/TMZ. Interestingly, 6 of the 7 patients with pseudoprogression in the CCNU/TMZ arm had a re-resection due to suspected progression which yielded a histology compatible with pseudoprogression (standard arm: 2/5 pseudoprogressions confirmed histologically).

\section{Postprogression therapy}

The median number of further lines of therapy was 2 (range 1-4) with TMZ and 1 (range 1-4) with CCNU/TMZ (for details, see Suppl. Table 4). While the overall rate of re-operations was higher after CCNU/TMZ (22.2\% vs. $30.3 \%)$, complete resections at progression were performed with similar frequency $(7.9 \% \mathrm{TMZ}$ vs. $9 \%$ CCNU/TMZ) but re-biopsies were exclusively performed after CCNU/TMZ (4.5\%). 
Re-RT was applied with similar frequency (23.8\% TMZ; $18.2 \%$ CCNU/TMZ). The rate of patients receiving any form of systemic antitumor therapy was higher after TMZ (60.3\% vs. $48.5 \%$; for details see Suppl. Table 4). Bevacizumab was applied with similar frequency (27\% TMZ, 30.3\% CCNU/TMZ).

\section{Toxicity, quality of life and neurocognition}

Table 2 summarizes AEs observed until 30 days after end of study therapy. There was no toxic death. The rate of patients with AEs grade $3 / 4$ was higher with CCNU/TMZ (all 59.1\%, hematotoxicity 36.4\%) than with TMZ (all 50.8\%, hematotoxicity $28 \cdot 6 \%$ ). Infectious complications were not increased with CCNU/TMZ. Regarding CNS AEs, there was a more frequent reporting of brain edema with CCNU/TMZ. The rate of some CNS symptoms such as speech impairment and sensory dysfunction were moderately increased in the experimental arm. The rate of nausea was increased from $19 \%$ with TMZ alone to $30 \%$ with CCNU/TMZ without an increase of vomiting. Low-grade alopecia was more frequent after CCNU/TMZ (27\% vs. $16 \%$ ). There was no excess of other non-hematologic, non-CNS organ toxicity in the CCNU/TMZ arm, in particular, there was no additional liver toxicity (Table 2). All patients were also evaluated for AEs reported for a minimum of 2 years after randomization (Supplementary Table 5), i.e. far beyond the end of study treatment. The extended observation period, potentially confounded by further lines of therapy, provided data on infrequent (3-6.1\% of patients) vascular events in the CCNU/TMZ arm: 4 patients with pulmonary embolism occurring at least four months after completion of study therapy were noted in the experimental arm only, although the rate of deep venous thromboses was not substantially different between the arms. Two patients with CNS hemorrhage (1 subdural, 1 epidural hematoma) had this AE 
during CCNU/TMZ therapy, another patient had a tumor hemorrhage later during bevacizumab therapy. Three patients had an ischemic stroke, 1 of them during CCNU/TMZ therapy (Table 2), 2 of them substantially later and after having received bevacizumab therapy. The longitudinal analysis of QoL and neurocognitive testing did not reveal systematic differences between the treatment arms. Detailed results will be presented in a separate publication. 


\section{Discussion}

The CeTeG/NOA-09 results provide evidence that CCNU/TMZ therapy may be superior to standard TMZ in patients with newly diagnosed MGMT promotormethylated glioblastoma. With CCNU/TMZ, OS was prolonged in the context of well tolerable toxicity. This may be a first step to improve drug therapy of glioblastoma beyond TMZ monotherapy and could be the initiation of separating drug therapy for MGMT-methylated from therapy of MGMT-nonmethylated glioblastoma.

As a limitiation, the CeTeG/NOA-09 was a small trial compared with previous phase III trials. ${ }^{1-3,5-8}$ A small number of patients are bearing the effect leading to significant survival differences between the treatment groups. This makes made the CeTeG/NOA-09 results more susceptible to confounding factors. For this reason, the planning of the trial already tried to anticipate potential imbalances of prognostic factors and to minimize their influence by using a test stratified for center and RPA class. An analysis stratified for RPA class accounts for known strong prognostic factors (KPS, age, extent of resection) since they are constitutive for the compound parameter RPA class. Nevertheless, the prespecified stratified log-rank test leads to small strata so that imbalances may have substantial influence on the results. For example, the definition of the stratified log-rank test statistic implies that patients from strata without matching patients in the other treatment arm do not contribute to the log-rank analysis. This is a very consequent way to enable valid comparisons. However, this characteristic of the stratified log-rank test led to the exclusion of some patients $(n=20)$ from the analysis. Under these circumstances, the Cox regression model as another prespecified analysis of the primary endpoint OS becomes very 
important. The multivariable Cox regression model (HR 0.60; 95\% Cl 0.35-1.03; $p=0.0641$ ) largely confirmed the finding of the stratified log-rank analysis of an OSprolonging effect in the CCNU/TMZ arm. Importantly, the stratified Cox regression model did not exclude any patients from the analysis and takes into account the problems with the covariates center and RPA class brought in by the small sample size. The treatment effect is further supported by exploratory analyses (Suppl. Fig 1b with inverse probability weighting; Suppl. Fig. 1c with analysis of the 14 centers without a lack of RPA3/RPA5 patients in the standard arm). Overall, the presence of an OS-prolonging effect is well supported by the fact that the predefined primary analysis was positive and by corroborating statistial analyses. Nevertheless, the results favouring CCNU/TMZ have to be taken with caution and conclusions have to consider the above mentioned limitations of the stratified analysis and the results of the unstratified analysis (Suppl. Fig. 1a).

As a further limitation, there is a discrepancy between prolonged OS and the lack of a difference in PFS. This discrepancy is not based on differences in further lines of therapy: re-irradiations and further chemotherapies were more frequent after TMZ; complete reresections and antiangiogenic treatments were similarly applied in both arms. An increased rate of late and prolonged pseudoprogressions after CCNU/TMZ could may have played a major role. Late and prolonged pseudoprogressions which, by definition, would have remained undetected by RANO criteria have already been described after CCNU/TMZ. ${ }^{27}$ The observation that most of the pseudoprogressions with CCNU/TMZ (6/7 vs. 2/5 with TMZ) were defined only by histology would be in line with this hypothesis. One could even hypothesize that undetected pseudoprogression was particularly prevalent in the first 2 years after the start of therapy thus providing an explanation for the observation that the PFS curves start 
separating late after 2 years (Fig. 3c). Future studies will have to analyze this phenomenon prospectively. Another influencing factor for the lack of a clear PFS signal would be the comparably small size of the trial which made the detection of small PFS differences less likely. One could speculate whether the observed OS prolongation may be in part due to long-term effects of CCNU as already described for lower grade tumors. ${ }^{30}$ Finally, the PFS graph (Fig. 3b) with a late separation of curves could also suggest that, regarding PFS, there may be two populations, one with and one without an additional benefit brought by combined CCNU/TMZ therapy. It will be interesting to see whether there are molecular differences between the tumors in these two groups.

Interestingly, median survival in the TMZ standard arm of CeTeG (31.4 months, 95\% $\mathrm{Cl} 27 \cdot 7-47 \cdot 1$ months) is prolonged as compared to historical controls of patients with a MGMT promotor-methylated tumors $(26.4$ months, $95 \% \mathrm{Cl} 23.9-34.7$ months, CENTRIC trial ${ }^{6}, 21.7$ months, $95 \% \mathrm{Cl}$ not supplied, TMZ registration trial $\left.{ }^{19}\right)$. Also, the 2 year survival rate was higher in the standard arm of CeTeG/NOA-09 $(69 \%, 95 \% \mathrm{Cl}$ $58-83 \%)$ as compared to the CENTRIC trial $(56 \%, 95 \% \mathrm{Cl} 49-62 \%) .{ }^{6}$ These modest differences between CeTeG/NOA-09 and CENTRIC, another trial for newly diagnosed glioblastoma patients with recruitment restricted to patients having a MGMT-promotor methylated tumor (same MGMT test and identical cutoff values), may not be accounted for by improvements in further line therapies since the portfolio of available therapies did not change between CENTRIC and CeTeG except for the availability of tumor-treating fields (TTF). TTF, however, had not been applied to any patient in the CeTeG trial. However, part of the differences may be explained by the particularly high rate of completely resected patients $(61 \% ;>95 \%$ with any type of resection) and patients with high performance score (82\% with KPS 90+) in 
CeTeG/NOA-09. Also, age was restricted to $<70$ years in CeTeG but not CENTRIC. These features indicate that the results of the $\mathrm{CeTeG/NOA}$ trial cannot be readily extrapolated and generalized to an unselected patient population. Also, it would be straightforward to apply the results to patients with an at least partially resected tumor and/or with a very high KPS although it has to be kept in mind that the subgroups of patients with biopsy only and lower KPS are too small to allow a meaningful subgroup analysis. In terms of generalization, it also has to be kept in mind that due to limitations of the methylation-specific PCR (MS-PCR) ${ }^{31}$ many neurooncological centers nowadays use pyrosequencing ${ }^{32,33}$ for the determination of the MGMT promotor methylation status. Nevertheless, the quantitative methylation-specific PCR $(\mathrm{MS}-\mathrm{PCR})^{22}$ applied here was the method of choice for many large randomized glioma trials $2,6,7,23,34$ and was the only certified method for MGMT promotor methylation analysis at the time when CeTeG/NOA-09 started.

As another interesting feature of CeTeG/NOA-09, the OS curves of TMZ and CCNU/TMZ separate late, about 2 years after randomization. This has not been observed in the TMZ registration trial ${ }^{1}$ and the EF14 trial (tumor-treating fields) ${ }^{2}$ but is well known from trials with $1 p / 19 q-$ codeleted anaplastic oligodendroglioma. ${ }^{10,11}$ One could speculate whether there are, in fact, two populations, one with additional benefit CCNU/TMZ and one without. Further analyses will have to look into associated molecular markers.

Toxicity of CCNU/TMZ was well acceptable and only in few domains (e.g. hematotoxicity) moderately increased compared with TMZ (Table 2). Classical CCNU-associated organ toxicities such as hepatopathy were not observed. The vascular AEs occuring predominantly late and with a long interval after termination of 
first-line therapy (ischemic stroke, pulmonary embolism, see Supplementary Material) have to be noted and future cohorts of CCNU/TMZ-treated patients should be systematically and prospectively analyzed for such events. Here, a particular interest should be put on the potential relationship with supportive therapy such as steroids and further lines of antitumor therapy, especially bevacizumab, both known to increase the rate of vascular AEs.

In conclusion, the data of the CeTeG trial demonstrated an OS benefit for CCNU/TMZ in the context of moderate toxicity. Therefore, CCNU/TMZ may be a promising therapeutic option for patients with MGMT promotor-methylated glioblastoma (age $<70$ ). The OS-positive CeTeG/NOA-09 trial provides a paradigm for molecular subgroup-specific therapy of glioblastoma and further optimization of combination chemotherapy for patients with MGMT-methylated glioblastoma. 


\section{Contributors}

UH, MS, MC, RF, CC, MG were responsible for the design of the trial. UH, TT, FM, JPS, US, MS, PH, RDK, DK, OG, RG, OS, OB, MU, CS, GT, TK, FR, FSG, BS, SB, AW, MR, LB, NG, $\mathrm{PV}, \mathrm{MM}, \mathrm{HV}, \mathrm{MS}, \mathrm{NS}, \mathrm{SK}, \mathrm{JW}, \mathrm{CS}, \mathrm{WS}, \mathrm{JCT}, \mathrm{MS}, \mathrm{MG}$ contributed to patient recruitment, treatment and data collection. MC, CC provided administrative support, reference neuropathology was performed by TP and reference neuroradiology was performed by VK, $\mathrm{MN}, \mathrm{HU}, \mathrm{EH}$. Data analysis and writing of the first drafts was performed by UH, TT, NS, JW, WW, MW, RF, MS, CC, MG. All authors approved the final version of the manuscript.

\section{Declaration of Interests}

UH reports grants and personal fees from Roche, personal fees and non-financial support from Medac, Bristol-Myers Squibb, personal fees from Novocure, Novartis, Daichii-Sankyo, Riemser, Noxxon. JPS reports a grant from Merck, personal fees and other support from Roche, Medac, Brisol-Myers Squibb, personal fees from Boehringer, Mundipharma. US reports other support from Medac, Schering Plough, Roche, Novocure, GSK, Novartis. PH reports personal fees from Medac, Bristol-Myers Squibb, Novocure, Merck. GT reports personal fees from Bristol-Myers Squibbs, Novocure, Medac. LB reports personal fees and non-financial support from Bristol-Myers Squibb, personal fees from Novartis, Jazz Pharmaceuticals, Pfizer, grants and personal fees from Sanofi, non-financial support from Amgen. NS reports personal fees and other support from Roche. CS reports personal fees from Roche. MC reports a grant from Gruenenthal. WW has a patent IDH antibody with royalties paid, a patent AHR diagnostics licensed, a patent Parvovirus issued, and a patent APG diagnostics licensed. MW reports grants and personal fees from Abbvie, Actelion, MSD, Novocure, Merck (EMD), Roche, grants from Acceleron, Bayer, OGD2, Piqur, Tragara, personal fees from BMS, Celgene, Celldex, Orbus, Progenics, Tocagen. MG reports grants, personal fees and other support from Medac, personal fees from Merck. All other authors have nothing to disclose. 


\section{Acknowledgement}

We thank patients and relatives for their participation and support. We are grateful to all local investigators and study nurses in the participating centers. We are particularly indebted to the excellent trial support and project management of the Clinical Study Core Unit, Study Center Bonn, especially by Yvonne Borck (Trial Center Bonn (SZB) and by Christiane Landwehr and Katja Rasch both Division of Clinical Neurooncology, University of Bonn Medical Center. We thank Gerald Illerhaus, Rita Engenhart-Cabillic and Martin Hellmich for their work in the independent data and safety monitoring board. This trial was supported by Clinical Trials Centre Cologne (CTCC), Medical Faculty, University of Cologne by performing Data Base Development, Data Management, Monitoring and Safety Management. We are very thankful to Meinhard Kieser (IMBI, University of Heidelberg) for performing a review of the statistical methods and calculations and to Martin van den Bent for critical reading and valuable discussion. The CeTeG/NOA-09 as an investigator-initiated trial was very generously funded by the German Federal Ministry of Education and Research (01KG1005). The investigators are very grateful for this support. 


\section{References}

1. Stupp R, Mason WP, van den Bent MJ, et al. Radiotherapy plus concomitant and adjuvant temozolomide for glioblastoma. N Engl J Med 2005;352:987-96.

2. Stupp R, Taillibert S, Kanner A, et al. Effect of Tumor-Treating Fields Plus Maintenance Temozolomide vs Maintenance Temozolomide Alone on Survival in Patients With Glioblastoma: A Randomized Clinical Trial. JAMA 2017;318: 230616.

3. Gilbert MR, Wang M, Aldape KD, et al. Dose-dense temozolomide for newly diagnosed glioblastoma: a randomized phase III clinical trial. J Clin Oncol 2013;31:4085-91.

4. Quinn JA, Jiang SX, Reardon DA, et al. Phase II trial of temozolomide (TMZ) plus irinotecan (CPT-11) in adults with newly diagnosed glioblastoma multiforme before radiotherapy. J Neuro-Oncol 2009;95:393-400.

5. Chinot OL, Wick W, Mason W, et al. Bevacizumab plus radiotherapytemozolomide for newly diagnosed glioblastoma. N Engl J Med 2014;370:709-22.

6. Stupp R, Hegi ME, Gorlia T, et al. Cilengitide combined with standard treatment for patients with newly diagnosed glioblastoma with methylated MGMT promoter (CENTRIC EORTC 26071-22072 study): a multicentre, randomised, open-label, phase 3 trial. Lancet Oncol 2014;15:1100-8.

7. Weller M, Butowski N, Tran DD, et al. Rindopepimut with temozolomide for patients with newly diagnosed, EGFRvlll-expressing glioblastoma (ACT IV): a randomised, double-blind, international phase 3 trial. Lancet Oncol 2017;18:1373-85. 
8. Walker MD, Green SB, Byar DP, et al. Randomized comparisons of radiotherapy and nitrosoureas for the treatment of malignant glioma after surgery. $N$ Engl $J$ Med 1980;303:1323-9.

9. Weller M, Muller B, Koch R, Bamberg M, Krauseneck P, Neuro-Oncology Working Group of the German Cancer S. Neuro-Oncology Working Group 01 trial of nimustine plus teniposide versus nimustine plus cytarabine chemotherapy in addition to involved-field radiotherapy in the first-line treatment of malignant glioma. J Clin Oncol 2003;21:3276-84.

10. Cairncross G, Wang M, Shaw E, et al. Phase III trial of chemoradiotherapy for anaplastic oligodendroglioma: long-term results of RTOG 9402. J Clin Oncol 2013;31:337-43.

11.van den Bent MJ, Brandes AA, Taphoorn MJ, et al. Adjuvant procarbazine, lomustine, and vincristine chemotherapy in newly diagnosed anaplastic oligodendroglioma: long-term follow-up of EORTC brain tumor group study 26951. J Clin Oncol 2013;31:344-50.

12. Wick W, Gorlia T, Bendszus M, et al. Lomustine and bevacizumab in progressive glioblastoma. N Engl J Med 2017;377:1954-1963.

13. Kohn KW. Interstrand cross-linking of DNA by 1,3-bis(2-chloroethyl)-1nitrosourea and other 1-(2-haloethyl)-1-nitrosoureas. Cancer Res $1977 ; 37: 1450-4$.

14. Wheeler GP, Bowdon BJ, Struck RF. Carbamoylation of amino acid, peptides, and proteins by nitrosoureas. Cancer Res 1975;35:2974-84 .

15. Plowman J, Waud WR, Koutsoukos AD, Rubinstein LV, Moore TD, Grever MR. Preclinical antitumor activity of temozolomide in mice: efficacy against human 
brain tumor xenografts and synergism with 1,3-bis(2-chloroethyl)-1-nitrosourea. Cancer Res 1994;54:3793-9.

16. Barrié M, Couprie C, Dufour $\mathrm{H}$,et al. Temozolomide in combination with BCNU before and after radiotherapy in patients with inoperable newly diagnosed glioblastoma multiforme. Ann Oncol 2005; 16:1177-84

17. Herrlinger U, Rieger J, Koch D, et al. Phase II trial of lomustine plus temozolomide chemotherapy in addition to radiotherapy in newly diagnosed glioblastoma: UKT-03. J Clin Oncol 2006;24:4412-7.

18. Glas M, Happold C, Rieger J, et al. Long-term survival of patients with glioblastoma treated with radiotherapy and lomustine plus temozolomide. J Clin Oncol 2009;27:1257-61.

19. Hegi ME, Diserens AC, Gorlia T, et al. MGMT gene silencing and benefit from temozolomide in glioblastoma. N Engl J Med 2005;352:997-1003.

20. Jakacki RI, Cohen KJ, Buxton A, et al. Phase 2 study of concurrent radiotherapy and temozolomide followed by temozolomide and lomustine in the treatment of children with high-grade glioma: a report of the Children's Oncology Group ACNS0423 study. Neuro Oncol 2016;18:1442-50.

21. Dupont WD and Plummer WD. PS power and sample size program available for free on the Internet. Controlled Clin Trials 1997; 18:274

22. Vlassenbroeck I1, Califice S, Diserens AC, et al. Validation of real-time methylation-specific PCR to determine O6-methylguanine-DNA methyltransferase gene promoter methylation in glioma. J Mol Diagn $2008 ; 10: 332-7$

23. Herrlinger U, Schafer N, Steinbach JP, et al. Bevacizumab plus irinotecan versus temozolomide in newly diagnosed O6-methylguanine-DNA methyltransferase 
nonmethylated glioblastoma: the randomized GLARIUS trial. J Clin Oncol 2016;34:1611-9.

24. Louis DN, Perry A, Reifenberger G, et al. The 2016 World Health Organization Classification of Tumors of the Central Nervous System: a summary. Acta Neuropathol 2016;131:803-20.

25. Capper D, Weissert S, Balss J, et al. Characterization of R132H mutation-specific IDH1 antibody binding in brain tumors. Brain Pathol 2010;20:245-54.

26. Wen PY, Macdonald DR, Reardon DA, et al. Updated response assessment criteria for high-grade gliomas: response assessment in neuro-oncology working group. J Clin Oncol 2010;28:1963-72

27. Stuplich M, Hadizadeh DR, Kuchelmeister K, et al. Late and prolonged pseudoprogression in glioblastoma after treatment with lomustine and temozolomide. J Clin Oncol 2012;30:e180-3

28. Mirimanoff RO, Gorlia T, Mason W, et al. Radiotherapy and temozolomide for newly diagnosed glioblastoma: Recursive partitioning analysis of the EORTC 26981/22981-NCIC CE3 phase III randomized trial. J Clin Oncol 2006;24:25639.

29. Louis DN, Ohgaki H, Wiestler OD, et al. The 2007 WHO classification of tumours of the central nervous system. Acta Neuropathol 2007;114:97-109.

30. Peyre M, Cartalat-Carel S, Meyronet D, et al. Prolonged response without prolonged chemotherapy: a lesson from PCV chemotherapy in low-grade gliomas. Neuro Oncol 2010;12:1078-82.

31. Quillien V, Lavenu A, Karayan-Tapon L, et al. Comparative assessment of 5 methods (methylation-specific polymerase chain reaction, MethyLight, 
pyrosequencing, methylation-sensitive high-resolution melting, and immunohistochemistry) to analyze O6-methylguanine-DNA-methyltranferase in a series of 100 glioblastoma patients. Cancer 2012;118:4201-11

32. Mikeska T, Bock C, El-Maarri O, et al. Optimization of quantitative MGMT promoter methylation analysis using pyrosequencing and combined bisulfite restriction analysis. J Mol Diagn 2007;9:368-81

33. Quillien V, Lavenu A, Ducray F, et al. Validation of the high-performance of pyrosequencing for clinical MGMT testing on a cohort of glioblastoma patients from a prospective dedicated multicentric trial. Oncotarget 2016;7:61916-29

34.van den Bent MJ, Baumert B, Erridge SC, et al. Interim results from the CATNON trial (EORTC study 26053-22054) of treatment with concurrent and adjuvant temozolomide for 1p/19q non-co-deleted anaplastic glioma: a phase 3 , randomised, open-label intergroup study. Lancet. 2017 Oct 7;390:1645-1653 


\section{Figures}

\section{Fig. 1: CONSORT diagram of the CeTeG trial}

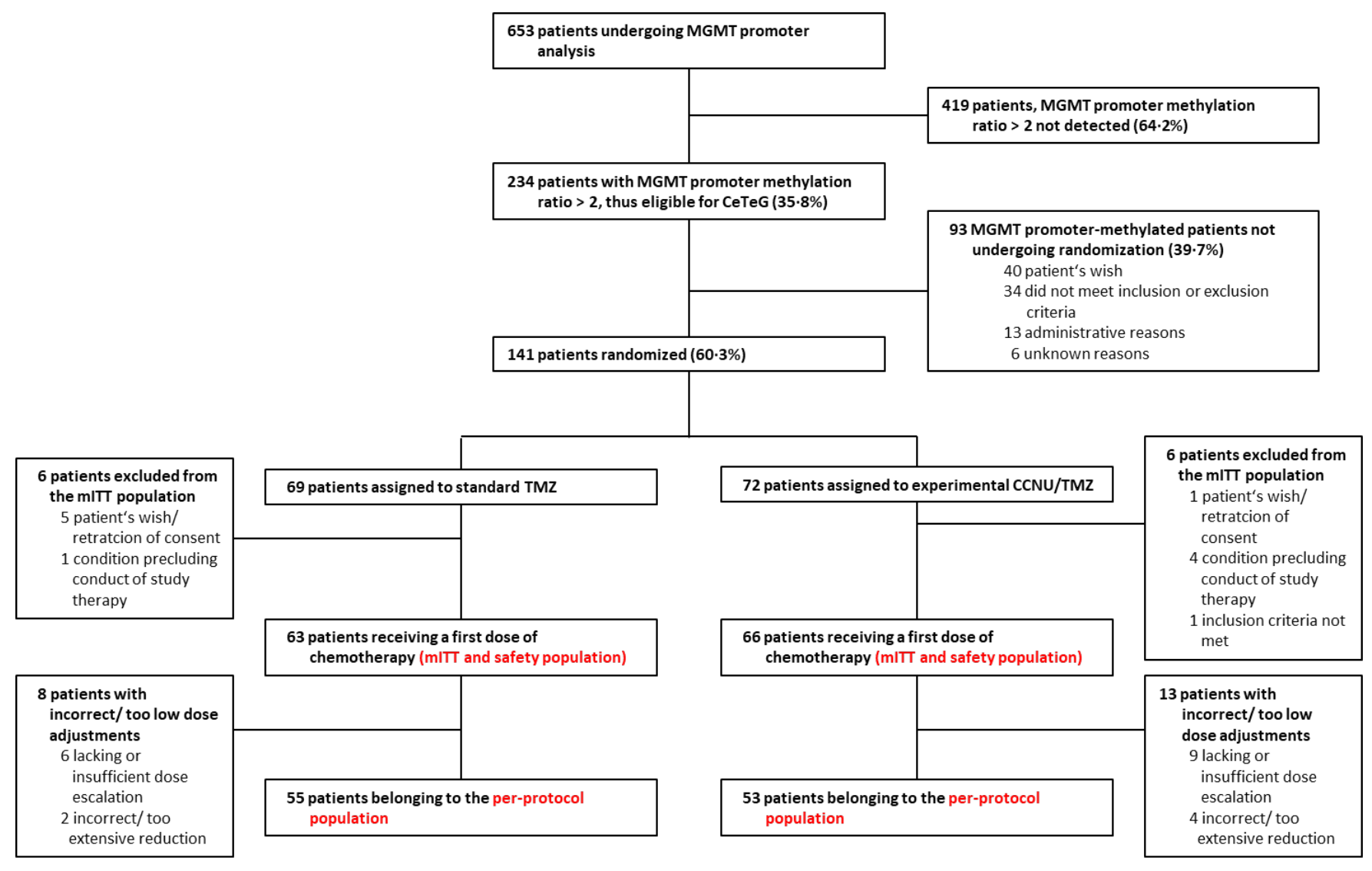




\section{Fig. 2: The CeTeG trial: schematic overview}

Arm A comprises the standard chemotherapy with temozolomide (TMZ). This included daily radiotherapy (RT)-concomitant temozolomide therapy $\left(75 \mathrm{mg} / \mathrm{m}^{2} / \mathrm{d}\right)$. Four weeks after the end of RT, the first of six adjuvant TMZ courses started (150 $\mathrm{mg} / \mathrm{m}^{2} / \mathrm{d} \mathrm{d} 1-5$ of a 28-day course). According to the standard and if no toxicity ensued, TMZ had to be escalated to $200 \mathrm{mg} / \mathrm{m}^{2} / \mathrm{d}$ in further courses (for further details on dose adaptations, see Patients and Methods)

In the experimental arm B, CCNU/TMZ therapy (CCNU $100 \mathrm{mg} / \mathrm{m}^{2} / \mathrm{d}$ d1 and TMZ $100 \mathrm{mg} / \mathrm{m}^{2} / \mathrm{d} \mathrm{d} 2-6$ ) was given in six 42-courses. The first course started on the first day of radiotherapy. Therefore, there was no extended daily concomitant chemotherapy in the experimental arm. In case no toxicity ensued (for details on dose adaptations, see Patients and Methods), TMZ had to be escalated stepwise $\left(120 \mathrm{mg} / \mathrm{m}^{2} / \mathrm{d}, 150 \mathrm{mg} / \mathrm{m}^{2} / \mathrm{d}\right)$ to a maximum daily dose of $200 \mathrm{mg} / \mathrm{m}^{2} / \mathrm{d}$ in further courses.

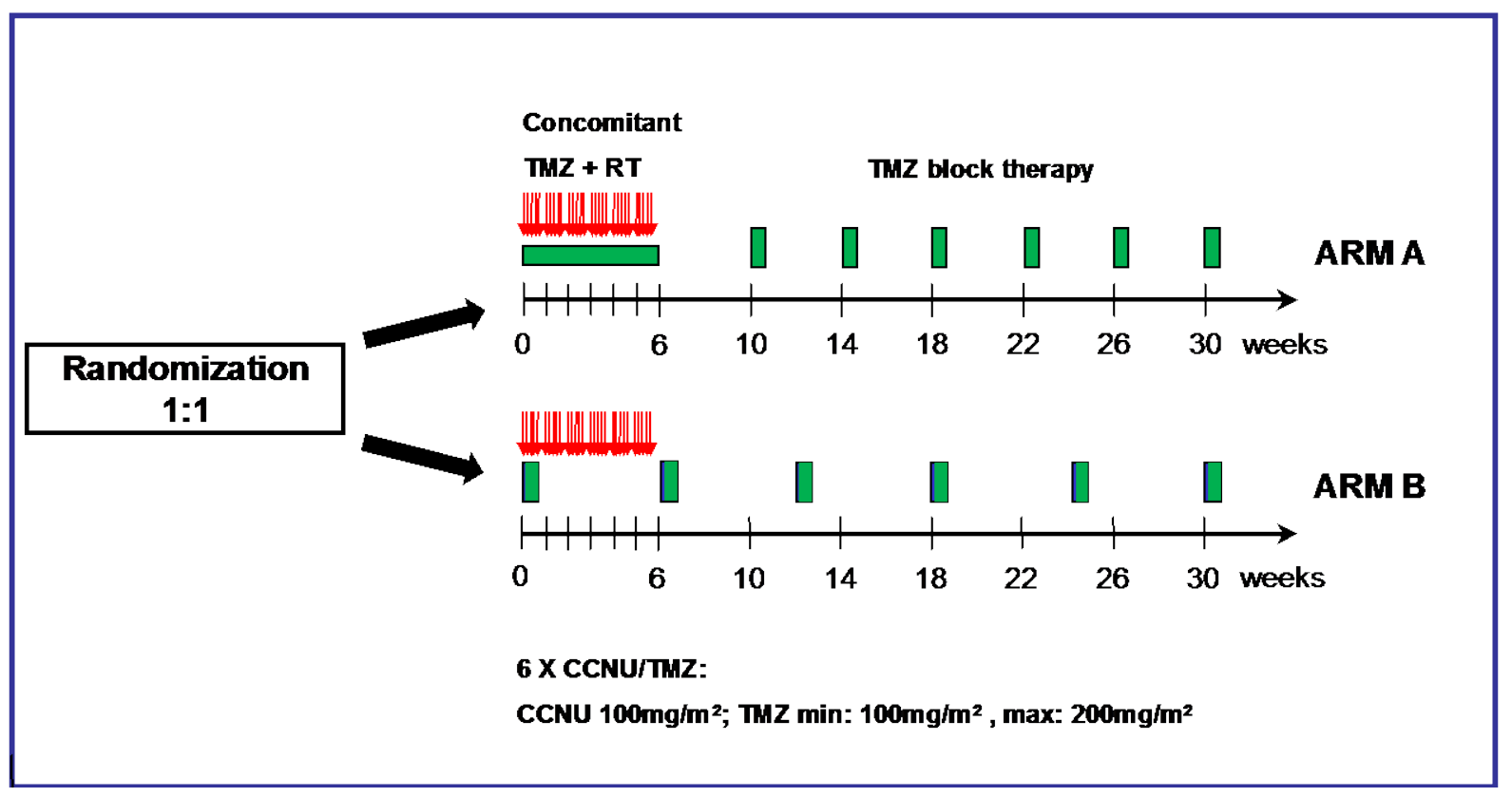


Fig. 3: Survival in the CeTeG/NOA-09 trial. Kaplan Meier graphs of patients with control counterparts in the respective center $x$ RPA class strata. Overall survival in (a) the mITT population ( $n=109 ; p=0.0492$ (stratified logrank test), HR $0.60(95 \% \mathrm{Cl}$ $0.35-1.03$ ) and (b) the as-randomized population (=ITT population; $n=125$; $\mathrm{p}=0.0432$ (stratified logrank test), HR $0.60(95 \% \mathrm{Cl} 0.35-1.03)$ ), progression-free survival in (c) the mITT population ( $n=109 ; p=0.4113$ (stratified logrank test), HR 0.91 $(95 \% \mathrm{Cl} 0.57-1.44)$ and (d) the as -randomized population (=ITT population, $\mathrm{n}=125$; $\mathrm{p}=0.4735$ (straified logrank test), HR 0.99 (95\% Cl 0.68-1.46).
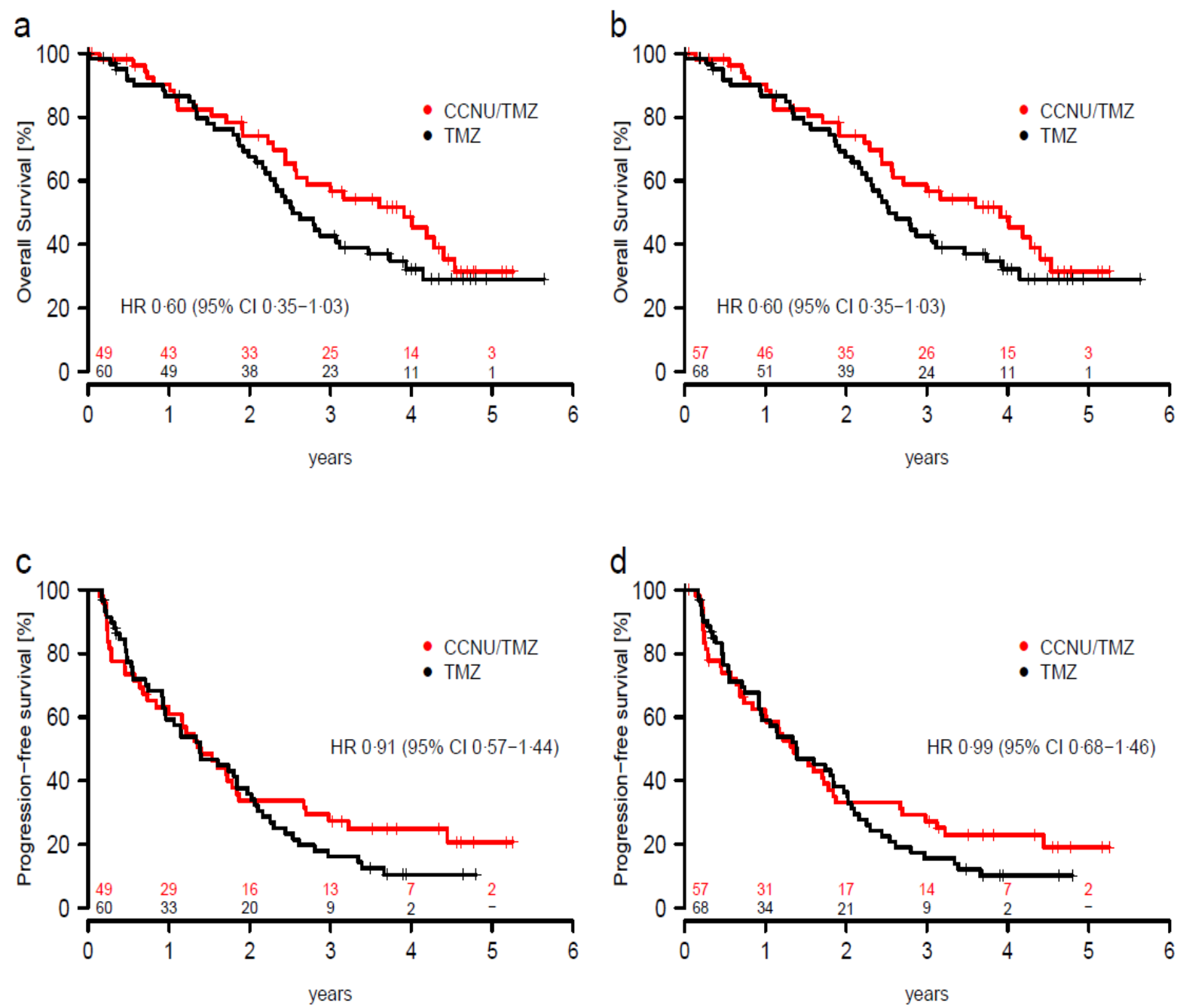
Tables

Table 1: Patient characteristics in the modified intent-to-treat population. P-values were computed using chi-squared tests for independence

\begin{tabular}{|c|c|c|c|c|}
\hline & $\begin{array}{c}\text { Total } \\
(n=129)\end{array}$ & $\begin{array}{c}\text { TMZ } \\
(n=63)\end{array}$ & $\begin{array}{c}\mathrm{CCNU/TMZ} \\
(\mathrm{n}=66)\end{array}$ & \\
\hline Gender, n (\%) & & & & $p=0.0074$ \\
\hline Male & $77(59 \cdot 7)$ & $30(47 \cdot 6)$ & $47(71 \cdot 2)$ & \\
\hline Female & $52(40 \cdot 3)$ & $33(52 \cdot 4)$ & $19(28 \cdot 8)$ & \\
\hline Median age, years (range) & & $56(28-70)$ & $59(31-71)$ & $p=0.21$ \\
\hline$<50$ years & $29(22 \cdot 5)$ & $11(17 \cdot 5)$ & $18(27 \cdot 3)$ & \\
\hline$\geq 50$ years & $100(77 \cdot 5)$ & $52(82.5)$ & $48(72 \cdot 7)$ & \\
\hline Initial KPS, n (\%) & & & & $p=0.25$ \\
\hline $90-100 \%$ & $106(82 \cdot 2)$ & $49(77 \cdot 8)$ & $57(86 \cdot 4)$ & \\
\hline $70-80 \%$ & $23(17 \cdot 8)$ & $14(22 \cdot 2)$ & $9(13 \cdot 6)$ & \\
\hline Not done & & & & \\
\hline
\end{tabular}

\section{Extent of resectiona, $\mathbf{n}(\%)$}

Stereotactic biopsy

Partial resection

Complete resection

$\begin{array}{ccc}4(3.1) & 1(1.6) & 3(4.5) \\ 46(35.7) & 22(34.9) & 24(36.4) \\ 79(61.2) & 40(63.5) & 39(59.1)\end{array}$

$\mathrm{p}=0.71$

MMSE, $\mathbf{n}(\%)$
$<27$
$\geq 27$
Missing
Molecular subgroup, $\mathbf{n}(\%)$
GBM IDHwt
GBM IDHmut
GBM-O
NA

$\begin{array}{ccc}19(14.7) & 8(12.7) & 11(16.7) \\ 108(83.7) & 55(87.3) & 53(80.3) \\ 2(1.6) & 0(0) & 2(3.0)\end{array}$

$p=0.62$

RPA group ${ }^{\mathrm{b}}, \mathrm{n}(\%)$

III

IV

$\mathrm{V}$

$\begin{array}{ccc}103(79.8) & 52(82 \cdot 5) & 51(77 \cdot 2) \\ 8(6 \cdot 2) & 5(7.9) & 3(4 \cdot 6) \\ 6(4 \cdot 7) & 3(4 \cdot 8) & 3(4 \cdot 6) \\ 12(9.3) & 3(4.8) & 9(13.6)\end{array}$

$\mathrm{p}=0.32$

aas determined by early ( $\leq 72 n)$ post-operative contrast-enhanced MRI; ${ }^{\circ R e c u r s i v e ~ p a r t i t i o n i n g ~ a n a l y s i s ~ g r o u p ~ a c c o r d i n g ~}$ to the modified EORTC classification ${ }^{28},{ }^{*} \mathrm{p}=0.007$, chi-square test,

Abbreviations: TMZ temozolomide, CCNU lomustine, KPS Karnofsky performance score, MMSE Mini mental state examination, GBM glioblastoma, IDH isocitrate dehydrogenase, GBM-O glioblastoma with oligodendroglial component, wt wildtype, mut mutated 
Table 2: Adverse events in the CeTeG trial until 30 days after end of study treatment (mITT population)

\begin{tabular}{|c|c|c|c|c|c|c|}
\hline \multirow{3}{*}{ No. of patients (\%) } & \multicolumn{3}{|c|}{ TMZ } & \multicolumn{3}{|c|}{ CCNU/TMZ } \\
\hline & \multicolumn{3}{|c|}{$(n=63)$} & \multicolumn{3}{|c|}{$(n=66)$} \\
\hline & All grades & Grade 3/4 & Grade 5 & All grades & Grade 3/4 & Grade 5 \\
\hline \multicolumn{7}{|l|}{ Hematotoxicity } \\
\hline Leukopenia & $10(15 \cdot 9)$ & $8(12 \cdot 7)$ & & $24(36 \cdot 4)$ & $10(15 \cdot 2)$ & \\
\hline Neutropenia & $7(11 \cdot 1)$ & $4(6 \cdot 3)$ & - & $12(18 \cdot 2)$ & $8(12 \cdot 1)$ & - \\
\hline Thrombocytopenia & $19(30 \cdot 2)$ & $15(23 \cdot 8)$ & & $40(60 \cdot 6)$ & $19(28 \cdot 8)$ & \\
\hline Lymphopenia & $4(6 \cdot 3)$ & $4(6 \cdot 3)$ & & $6(9 \cdot 1)$ & $3(4.5)$ & \\
\hline Anemia & $3(4 \cdot 8)$ & $3(4 \cdot 8)$ & & $5(7 \cdot 6)$ & $1(1 \cdot 5)$ & \\
\hline \multicolumn{7}{|l|}{ Infections } \\
\hline Upper Airways & $7(11 \cdot 1)$ & - & & $9(13 \cdot 6)$ & - & \\
\hline Lung & $4(6 \cdot 3)$ & $1(1 \cdot 6)$ & - & $2(3.0)$ & $2(3.0)$ & - \\
\hline Gastrointestinal & $1(1 \cdot 6)$ & - & & $3(4 \cdot 5)$ & $1(1.5)$ & \\
\hline Wound, other than CNS & $1(1 \cdot 6)$ & $1(1 \cdot 6)$ & & $3(4 \cdot 5)$ & $3(4 \cdot 5)$ & \\
\hline Fatigue & $14(22 \cdot 2)$ & - & & $17(25 \cdot 8)$ & - & \\
\hline \multicolumn{7}{|l|}{ Gastrointestinal } \\
\hline Nausea & $12(19 \cdot 0)$ & - & & $20(30 \cdot 3)$ & - & \\
\hline Vomiting & $8(12 \cdot 7)$ & - & & $6(9 \cdot 1)$ & - & \\
\hline Diarrhoea & $4(6 \cdot 3)$ & - & - & $2(3.0)$ & - & - \\
\hline Constipation & $12(19 \cdot 0)$ & - & & $15(22 \cdot 7)$ & - & \\
\hline Anorexia & $2(3 \cdot 2)$ & - & & $4(6 \cdot 1)$ & - & \\
\hline Weight loss & $1(1 \cdot 6)$ & - & & $2(3 \cdot 0)$ & - & \\
\hline Stomatitis & $1(1 \cdot 6)$ & - & & $4(6 \cdot 1)$ & - & \\
\hline \multicolumn{7}{|l|}{ Liver/Pancreas } \\
\hline Elevated transaminases & $4(6 \cdot 3)$ & - & & $3(4 \cdot 5)$ & - & \\
\hline Elevated gammaGT & - & - & - & $4(6 \cdot 1)$ & $4(6 \cdot 1)$ & - \\
\hline Elevated Bilirubine & $1(1 \cdot 6)$ & - & & - & - & \\
\hline Elevated Lipase & $2(3.2)$ & $1(1 \cdot 6)$ & & - & - & \\
\hline \multicolumn{7}{|l|}{ Cardial/Vascular } \\
\hline Arrhythmia & $2(3 \cdot 2)$ & - & & - & - & \\
\hline Hemorrhage & - & - & - & $2(3.0)$ & - & - \\
\hline Hypertension & - & - & & $1(1.5)$ & - & \\
\hline
\end{tabular}


Venous Thrombosis

Pulmonary embolism

\section{Neurological/psychiatrical}

Seizures

Ischemic stroke

CNS bleeding

Brain edema

Memory impairment

Motor dysfunction

Sensory dysfuntion

Speech impairment

Cognitive disturbance

Personality change

Anxiety

Sleeping problems

Incontinence

Hearing impariment

Dysgeusia

Dizziness

Wound problems CNS/Skull

Skin

Alopecia

Erythema

Exanthema/Rash

\section{Pain}

Headache

Radicular/peripheral nerve

Muscle

Joints
$16(25 \cdot 4) \quad 4(6 \cdot 3)$

$17(25 \cdot 8) \quad 6(9 \cdot 1)$

$1(1.5) \quad 1(1.5)$

$2(3.0)$

$9(13.6) \quad 2(3.0)$

$2(3.0)$

$8(12.1) \quad 2(3.0)$

$7(10.6) \quad 1(1.5)$

$9(13.6) \quad 3(4.5)$

$5(7 \cdot 6)$

$5(7 \cdot 6)$

$3(4.5)$

$5(7 \cdot 6)$

$1(1.5)$

$5(7 \cdot 6)$

$5(7 \cdot 6)$

$2(3.0)$

$1(1.5)$

$3(4 \cdot 8)$

$2(3 \cdot 2)$

$10(15.9) \quad 1(1.6)$

$18(27 \cdot 3) \quad 1(1.5)$

$2(3.2) \quad 1(1.6)$

$6(9 \cdot 1)$

$7(10 \cdot 6)$

$9(14 \cdot 3)$

$12(19 \cdot 0)$

$12(18 \cdot 1)$

2 (3.2)

$1(1.5)$

$2(3.0)$

$4(6 \cdot 1)$

Abbreviations: No. Number, TMZ temozolomide, CCNU lomustine 


\section{Supplementary Material (Web Appendix)}

\section{Phase III trial of CCNU/temozolomide (TMZ) combination therapy vs. standard TMZ therapy for newly diagnosed MGMT-methylated glioblastoma patients: the randomized, open- label CeTeG/NOA-09 trial}

Herrlinger et al. for the the Neurooncology Working Group (NOA) of the German Cancer Society 


\section{Supplementary Material: Methods}

\section{Trial oversight}

The sponsor of the trial was the University of Bonn. All sponsor-related duties were guided by standard operating procedures established at the Clinical Study Core Unit (Study Center Bonn) and the Center for Clinical Studies at the University of Cologne (ZKS Cologne). In this investigator-initiated trial, the principal investigator (PI, Ulrich Herrlinger) had the additional function of the sponsor-delegated person (SDP), which is in accordance with the Good Clinical Practice Guideline (ICH-E6; 1.54) and European law (regulation 536/2014 article 71). As designee of the sponsor, the $\mathrm{PI} / \mathrm{SDP}$ was responsible for all sponsor-related functions including the proper conduct of the trial according to the protocol, correct analysis and interpretation of the resulting data, and the communication with regulatory authorities. The study coordinator (Martin Glas) supported the PI/SDP in the communication with the different trial sites, especially concerning the medical treatment. To be able to perform all the sponsor duties in high quality, the PI/SDP was supported by the Clinical Study Core Unit (Study Center Bonn, Head Christoph Coch; biometry, project management, quality management) and the Center for Clinical Studies at the University of Cologne (ZKS Cologne; data management, monitoring, pharmacovigilance). All procedures associated with randomization, data acquisition and monitoring were carried out so that the PI/SDP could not interfere with these processes. The data acquisition and monitoring were performed by the Center for Clinical Studies at the University of Cologne and the randomization by the Clinical Study Core Unit at the Study Center Bonn. Thus, these essential processes were all performed at locations separated from the Bonn trial site at the Division of Clinical Neurooncology/Department of Neurology. 
The randomization list was generated using SAS at the Institute for Medical Biometry, Informatics and Epidemiology (IMBIE, Rolf Fimmers). The randomization was blocked with a block size of 6 and was stratified for center by allocating complete blocks to a center. The randomization list was kept at the Study Center Bonn without access for any external individual, including the PI/SDP and the clinical study team of the $\mathrm{PI}$ at the Division of Clinical Neurooncology. By fax, the trial sites contacted the team of Clinical Study Core Unit (Study Center Bonn) for randomization. After checking the in- and exclusion criteria listed on the randomization fax form, the trial site received a fax with the randomization details incl. the allocation to the treatment arm. Every randomization was documented, including the person performing the randomization at the Clinical Study Core Unit (Study Center Bonn). The trial site of the PI at the Division of Clinical Neurooncology had to undergo exactly the same ranodmization procedure for its patients as all other centers in the trial.

The PI and the leading statistician (Rolf Fimmers) had no access to the data of the trial until the export of the full data base from the ZKS Cologne to the IMBIE. This took place after the closure of trial and closure of the trial data bank, which prior to that had undergone final monitoring and quality checks. There were 2 exceptions to this rule: (1) Every 6 months until the end of the treatment phase of the last patient randomized, the ZKS Cologne provided a safety report with frequencies of serious adverse reactions and adverse event rates for the discussion in the independent Drug Monitoring and Safety Board meetings. (2) Fourteen months after last-patientin, a blinded survival analysis was performed to estimate the overall event rate in the whole study population. The leading statistician received survival data blinded for the treatment arm and the only information that the PI received was the event rate for 
death in the whole study population. This data point was only used to determine the prolongation of the follow-up time until final closure of the trial. After the delivery of the final database, the leading statistician statistician (RF) and the PI (together with TT, CC and MG) had full access to all aspects of the data. According to standard operating procedures, the primary endpoint analysis was performed by the leading statistician (RF) and a second calculation (yielding identical results) was carried out by another statistician at the IMBIE.

\section{Recruitment of patients, central reference neuropathology and central MGMT testing}

The recruitment of patients was a two-step process: In the first step taking place up to three weeks after operation/biopsy, patients were screened and informed consent was obtained for the central reference neuropathology (TP, Institute for Neuropathology, University of Bonn) and MGMT promotor methylation (MDX Health, Herstal, Belgium) testing as a study procedure. Paraffin-embedded tumor specimens were shipped to central reference neuropathology, and, only after confirmation of glioblastoma/gliosarcoma diagnosis, slides with confirmed tumor (position of the tumor marked on the slide for extraction) were sent to central MGMT analysis, both by overnight carrier. The whole process was documented and guided by standard operation procedures. Results of the MGMT promotor methylation analysis were directly reported to the centers (turnaround time from sending to neuropathology until reception of the MGMT results about 14 days). If then all other inclusion criteria applied incl. methylated MGMT promotor status, the informed consent for randomization and treatment within the trial was obtained and randomization using a fax form including the check of the inclusion/exclusion criteria ensued. 
Randomization could only be performed if the treatment could start within 35 days after operation/biopsy.

\section{Kaplan Meier plot with inverse probability weights}

The supplementary results include a Kaplan Meier plot for overall survival applying inverse probability weights. This technique up- or down-weights patients treated in a center with an RPA class distribution different from the one in the whole study population (factor $>1$ for patients underrepresented, factor $<1$ for patients overrepresented in this center). The calculation of a Kaplan Meier plot with inverse probability weights was based on 3 strata for RPA class (RPA class 3, 4, and 5) and 2 strata for center (1 stratum comprising the 40 patients treated in centers with no RPA3 or RPA5 patients in the standard arm and 1 stratum comprising the 89 patients treated in centers without this sort of imbalance). For a combination of stratifiers (RPA and center) containing in total $s=S_{\exp }+S_{c}$ patients (Sexp experimental CCNU/TMZ arm, sc control group) these patients obtained weights in the proportions $\mathrm{s} / 2 \times \mathrm{S}_{\exp }$ and $\mathrm{s} / 2 \times \mathrm{sc}_{\mathrm{c}}$, thus balancing for the over- or underrepresentation of one of the treatment groups in each stratum. 


\section{Supplementary Figure}

\section{Supplementary Figure 1: Overall survival in the CeTeG trial, post-hoc analyses}

(a) Kaplan-Meier graph for overall survival including all 129 patients of the mITT population (unstratified log-rank analysis $\mathrm{p}=0.6579$; univariate Cox regression analysis $\mathrm{HR} 0.90,95 \% \mathrm{Cl} 0.58-1.41)$. The median overall survival was prolonged from 31.4 months with $\mathrm{TMZ}(95 \% \mathrm{Cl} 27 \cdot 0-44.8$ months $)$ to 37.9 months with CCNU/TMZ (95\% Cl 29.2-51.4 months). (b) Kaplan-Meier graph for overall survival including data from all 129 patients of the mITT population and using inverse probability weights to account for the fact that in 3 centers of the trial all patients with RPA class 3 or 5 belonged to the experimental arm and none to the standard arm. Based on this graph, median overall survival was prolonged from 30.4 months (95\% Cl 27-44.9 months) in the TMZ arm to 46.9 months (95\% Cl 31-NA months) in the CCNU/TMZ arm. Also, the 4-year OS rate was increased from $31 \cdot 4 \%(20 \cdot 8-47 \cdot 5 \%)$ to $48.8 \%(37.5-63.5 \%)$ (c) Kaplan-Meier graph including 89 patients from all centers except for the 3 centers with only RPA class 4 (and no class 3 or 5 ) patients in the standard arm (stratified log-rank test $\mathrm{p}=0.0378$; multivariate Cox regression analysis with center and RPA class as covariates HR $0.53,95 \% \mathrm{Cl} 0.29-0.99)$. The median overall survival was prolonged from 29.4 months $(95 \% \mathrm{Cl} 23.9-34.4$ months) in the TMZ arm to 46.9 months (95\% $\mathrm{Cl} 29.3-54.5$ months) 
a

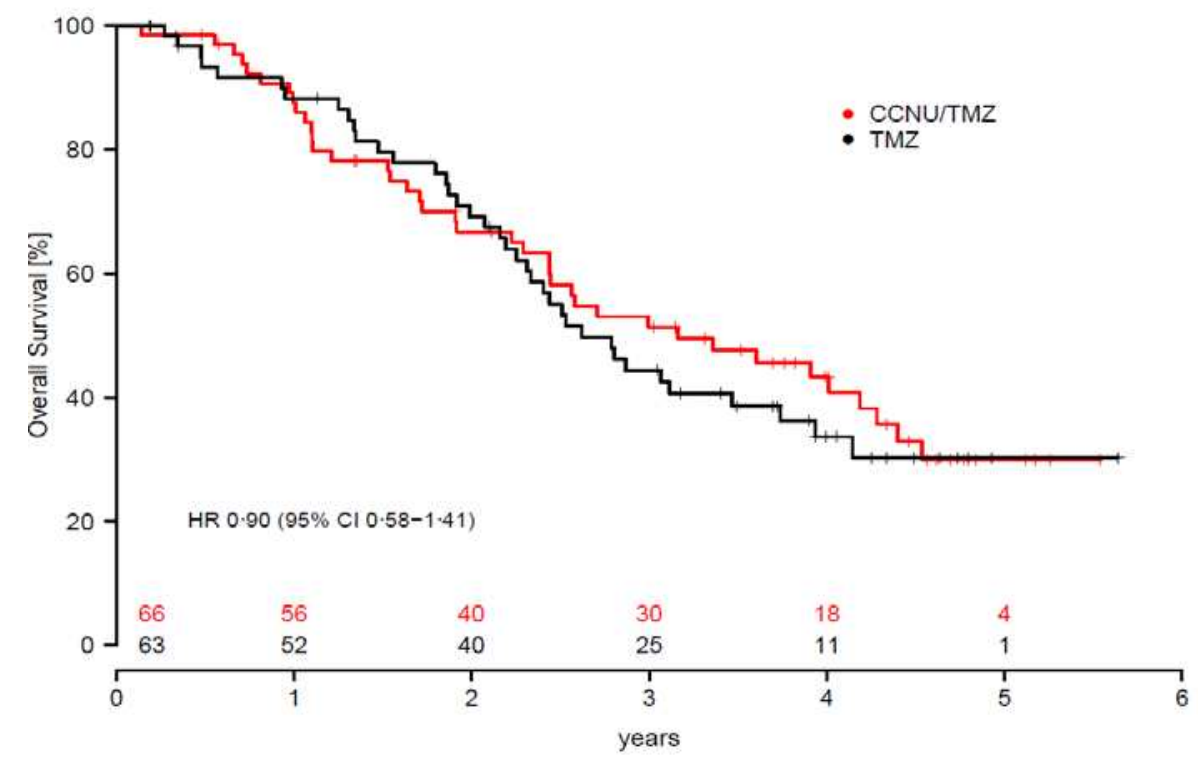

b

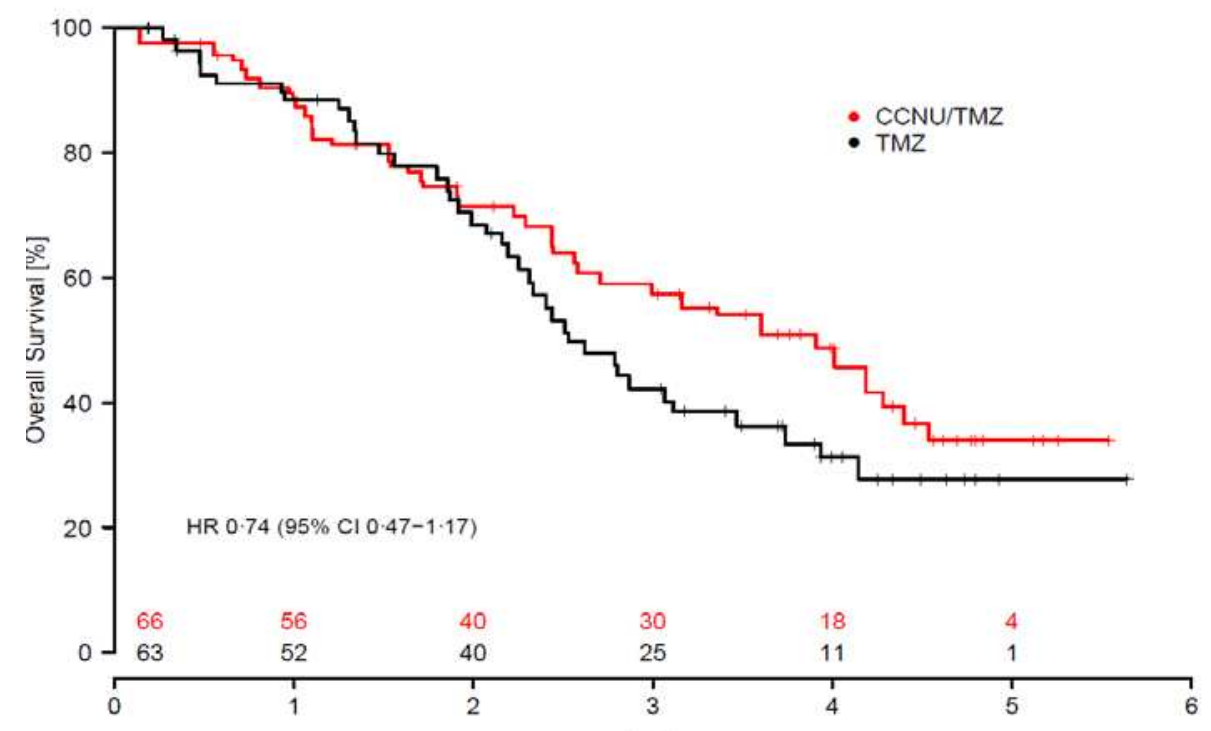

C

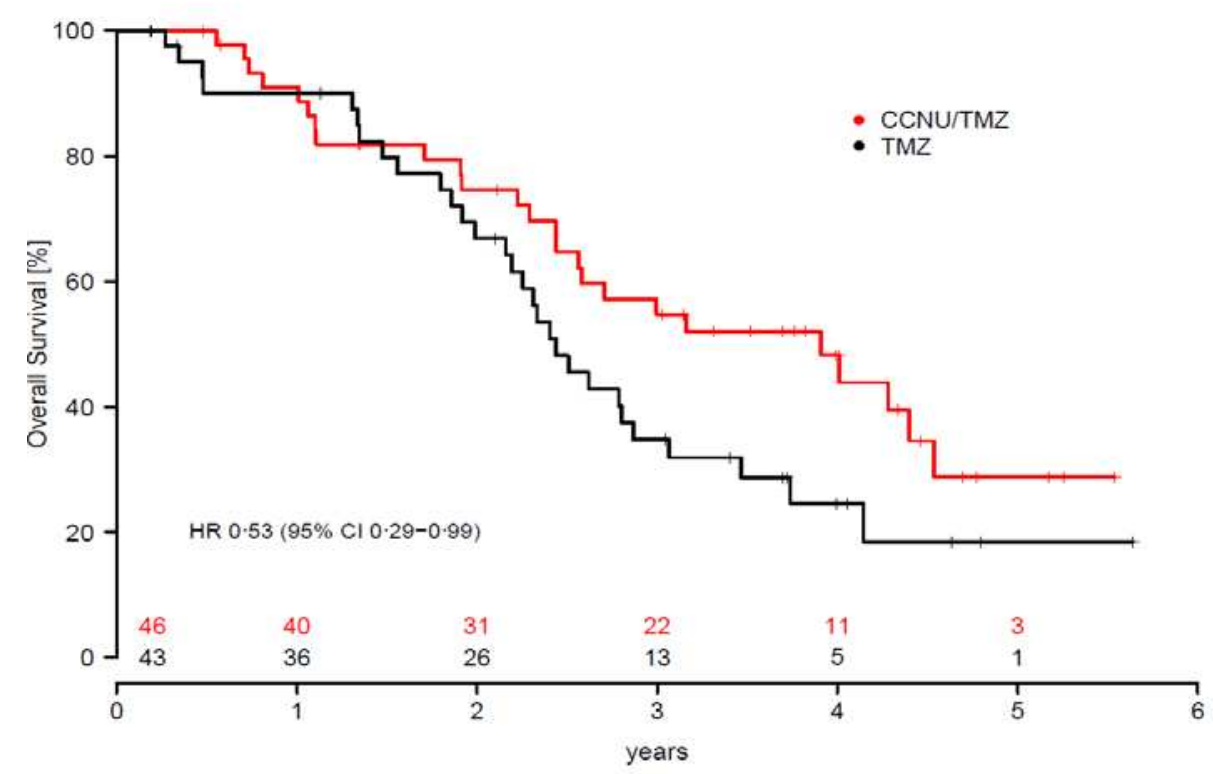




\section{Supplementary Tables}

Supplementary Table 1: Application of chemotherapy in the CeTeG trial (mITT population)

\begin{tabular}{|c|c|c|c|c|}
\hline & \multicolumn{2}{|c|}{$\begin{array}{r}\text { TMZ } \\
(n=63)\end{array}$} & \multicolumn{2}{|c|}{ CCNU/TMZ $(n=66)$} \\
\hline \multicolumn{5}{|l|}{$\begin{array}{l}\text { Median number of adjuvant } \\
\text { courses (range) }\end{array}$} \\
\hline & 6 & $(0-6)$ & 5 & $(1-6)$ \\
\hline \multicolumn{5}{|l|}{$\begin{array}{c}\text { Number of } \\
\text { courses started (N;\%) }\end{array}$} \\
\hline RT-concomitant therapy & 63 & 100 & - & - \\
\hline course 1 & 53 & $84 \cdot 1$ & 66 & 100 \\
\hline course 2 & 48 & $76 \cdot 2$ & 61 & 92.4 \\
\hline course 3 & 46 & 73 & 53 & $80 \cdot 3$ \\
\hline course 4 & 45 & $71 \cdot 4$ & 48 & $72 \cdot 7$ \\
\hline course 5 & 40 & 63.5 & 36 & $54 \cdot 6$ \\
\hline course 6 & 38 & 60.3 & 26 & 39.4 \\
\hline \multicolumn{5}{|c|}{ Maximal TMZ dose escalation $(\mathrm{N} ; \%)$} \\
\hline no adjuvant TMZ course & 10 & $15 \cdot 9$ & & \\
\hline $100 \mathrm{mg} / \mathrm{m}^{2}$ & 2 & 3.2 & 24 & $36 \cdot 4$ \\
\hline $120-125 \mathrm{mg} / \mathrm{m}^{2}$ & 2 & $3 \cdot 2$ & 12 & $18 \cdot 1$ \\
\hline $150 \mathrm{mg} / \mathrm{m}^{2}$ & 7 & $11 \cdot 1$ & 5 & $7 \cdot 6$ \\
\hline $200 \mathrm{mg} / \mathrm{m}^{2}$ & 42 & 66.7 & 25 & 37.9 \\
\hline
\end{tabular}




\begin{tabular}{|c|c|c|}
\hline \multicolumn{3}{|c|}{$\begin{array}{l}\text { Supplementary Table 2: Cumulative daily doses of chemotherapy applied (mITT } \\
\text { population) }\end{array}$} \\
\hline $\begin{array}{l}\text { Cumulative daily doses }\left(\mathrm{mg} / \mathrm{m}^{2} / \mathrm{day}\right) \text { per patient } \\
\text { during cycle therapy }\end{array}$ & TMZ & CCNU/TMZ \\
\hline $\mathrm{TMZ}(\mathrm{mg}$, mean \pm stdev) & $882+/-334$ & $489+/-261$ \\
\hline 25th percentile & 750 & 270 \\
\hline 50th percentile & 1000 & 470 \\
\hline 75th percentile & 1150 & 745 \\
\hline maximum & 1150 & 870 \\
\hline CCNU (mg, mean +/- stdev) & - & $351+/-139$ \\
\hline 25th percentile & - & 275 \\
\hline 50th percentile & - & 400 \\
\hline 75th percentile & - & 500 \\
\hline maximum & - & 540 \\
\hline
\end{tabular}




\begin{tabular}{|c|c|c|c|}
\hline \multicolumn{4}{|c|}{$\begin{array}{l}\text { Supplementary Table 3: Summary of further tumor therapy in both arms of the } \\
\text { CeTeG/NOA-09 trial (mITT population) }\end{array}$} \\
\hline & & $\begin{array}{l}\text { TMZ } \\
(n=63)\end{array}$ & $\begin{array}{l}\text { CCNU/TMZ } \\
(\mathrm{n}=66)\end{array}$ \\
\hline $\begin{array}{l}\text { Any further line } \\
\text { therapy }\end{array}$ & & $41(65 \cdot 1 \%)$ & $40(60 \cdot 1 \%)$ \\
\hline \multirow[t]{3}{*}{$\begin{array}{l}\text { No further therapy } \\
\text { documented }\end{array}$} & & $22(34 \cdot 9 \%)$ & $26(39 \cdot 4 \%)$ \\
\hline & No progressive disease, alive & $11(17 \cdot 5 \%)$ & $12(18 \cdot 2 \%)$ \\
\hline & Progressive disease or death & $11(17 \cdot 5 \%)$ & $14(21 \cdot 2 \%)$ \\
\hline Re-operation & & $15(23 \cdot 8 \%)$ & $20(30 \cdot 3 \%)$ \\
\hline Re-radiotherapy & & $15(23 \cdot 8 \%)$ & $12(18 \cdot 2 \%)$ \\
\hline \multirow[t]{10}{*}{$\begin{array}{l}\text { Any form of systemic } \\
\text { antitumor therapy }\end{array}$} & & $39(61.9 \%)$ & $33(50 \%)$ \\
\hline & TMZ monotherapy & $26(41 \cdot 3 \%)$ & $26(39 \cdot 4 \%)$ \\
\hline & CCNU monotherapy or procarbacine/CCNU & $10(15 \cdot 9 \%)$ & $3(4 \cdot 5 \%)$ \\
\hline & CCNU/TMZ & $3(4 \cdot 8 \%)$ & $1(1.5 \%)$ \\
\hline & Carboplatin/Etoposide & $0(0 \%)$ & $1(1.5 \%)$ \\
\hline & BEV monotherapy or in any combination with BEV & $18(28 \cdot 6 \%)$ & $21(31.8 \%)$ \\
\hline & $\mathrm{BEV}+/$ - irinotecan & $9(14 \cdot 3 \%)$ & $17(25 \cdot 8 \%)$ \\
\hline & BEV + any alkylating chemotherapy & $9(14 \cdot 3 \%)$ & $4(6 \cdot 1 \%)$ \\
\hline & Nivolumab & $1(1 \cdot 6 \%)$ & $1(1.5 \%)$ \\
\hline & Experimental multikinase inhibitor & $1(1 \cdot 6 \%)$ & $1(1 \cdot 5 \%)$ \\
\hline
\end{tabular}


Supplementary Table 4: Frequency of dose delays during the adjuvant chemotherapy courses (mITT population)

\begin{tabular}{|c|c|c|c|}
\hline Start of course & Delay & $\begin{array}{l}\text { TMZ arm } \\
\text { (\% of evaluable } \\
\text { patients) }\end{array}$ & $\begin{array}{l}\text { CCNU/TMZ arm } \\
\text { (\% of evaluable } \\
\text { patients) }\end{array}$ \\
\hline \multirow[t]{3}{*}{2} & $\begin{array}{l}\text { timely or less than } 2 \text { weeks } \\
\text { delay }\end{array}$ & 92 & $82 \cdot 5$ \\
\hline & delay 2-6 weeks & $6 \cdot 0$ & $14 \cdot 3$ \\
\hline & delay more than 6 weeks & $2 \cdot 0$ & $3 \cdot 2$ \\
\hline \multirow[t]{3}{*}{3} & $\begin{array}{l}\text { timely or less than } 2 \text { weeks } \\
\text { delay }\end{array}$ & $80 \cdot 9$ & $83 \cdot 3$ \\
\hline & delay 2-6 weeks & $17 \cdot 0$ & $13 \cdot 0$ \\
\hline & delay more than 6 weeks & $2 \cdot 1$ & 3.7 \\
\hline \multirow[t]{3}{*}{4} & $\begin{array}{l}\text { timely or less than } 2 \text { weeks } \\
\text { delay }\end{array}$ & $77 \cdot 8$ & $75 \cdot 5$ \\
\hline & delay 2-6 weeks & $6 \cdot 7$ & $22 \cdot 4$ \\
\hline & delay more than 6 weeks & 0.0 & $2 \cdot 0$ \\
\hline \multirow[t]{3}{*}{5} & $\begin{array}{l}\text { timely or less than } 2 \text { weeks } \\
\text { delay }\end{array}$ & 80.5 & $55 \cdot 3$ \\
\hline & delay 2-6 weeks & $17 \cdot 1$ & 39.5 \\
\hline & delay more than 6 weeks & $2 \cdot 4$ & $5 \cdot 3$ \\
\hline \multirow[t]{3}{*}{6} & $\begin{array}{l}\text { timely or less than } 2 \text { weeks } \\
\text { delay }\end{array}$ & $76 \cdot 3$ & $45 \cdot 2$ \\
\hline & delay 2-6 weeks & $21 \cdot 1$ & 38.7 \\
\hline & delay more than 6 weeks & $2 \cdot 6$ & $16 \cdot 1$ \\
\hline
\end{tabular}

Abbreviations: RT radiotherapy, TMZ temozolomide, CCNU lomustine 


\begin{tabular}{|c|c|c|c|c|c|c|}
\hline \multicolumn{7}{|c|}{$\begin{array}{l}\text { Supplementary Table 5: Adverse events in the CeTeG trial until at least } 2 \text { years } \\
\text { after end of study treatment } \\
\text { (mITT population) }\end{array}$} \\
\hline \multirow{3}{*}{$\begin{array}{l}\text { Number of patients } \\
(\%)\end{array}$} & \multirow{2}{*}{\multicolumn{3}{|c|}{$\begin{array}{l}\text { TMZ } \\
(n=63)\end{array}$}} & \multirow{2}{*}{\multicolumn{3}{|c|}{$\begin{array}{l}\text { CCNU/TMZ } \\
(n=66)\end{array}$}} \\
\hline & & & & & & \\
\hline & All grades & Grade $3 / 4$ & Grade 5 & All grades & Grade 3/4 & Grade 5 \\
\hline \multicolumn{7}{|l|}{ Cardial/Vascular } \\
\hline Arrhythmia & $3(4 \cdot 8)$ & $1(1 \cdot 6)$ & & - & - & \\
\hline Hemorrhage & - & - & - & $2(1.5)$ & - & - \\
\hline Hypertension & $3(4 \cdot 8)$ & - & & $2(3 \cdot 0)$ & $1(1.5)$ & \\
\hline Venous thrombosis & $3(4 \cdot 8)$ & $2(3 \cdot 2)$ & & $3(4 \cdot 8)$ & $1(1.5)$ & \\
\hline Pulmonary embolism & - & - & & $4(6 \cdot 1)$ & $3(4 \cdot 5)$ & \\
\hline \multicolumn{7}{|l|}{$\begin{array}{l}\text { Neurological/ } \\
\text { psychiatric }\end{array}$} \\
\hline Seizures & $21(33 \cdot 3)$ & $9(14 \cdot 3)$ & & $24(36 \cdot 4)$ & $10(15 \cdot 2)$ & $1(1.5)$ \\
\hline Ischemic stroke & - & - & & $3(4 \cdot 5)$ & $2(3 \cdot 0)$ & \\
\hline CNS bleeding & - & - & & $3(4 \cdot 5)$ & - & \\
\hline Brain edema & $4(6 \cdot 3)$ & $2(3 \cdot 2)$ & & $9(13 \cdot 6)$ & $2(3 \cdot 0)$ & \\
\hline Memory impairment & $1(1 \cdot 6)$ & - & & $2(3 \cdot 0)$ & - & \\
\hline Motor dysfunction & $16(25 \cdot 4)$ & $6(9.5)$ & & $16(24 \cdot 2)$ & $4(6 \cdot 1)$ & \\
\hline Sensory dysfuntion & $3(4 \cdot 8)$ & - & & $9(13 \cdot 6)$ & $1(1.5)$ & \\
\hline Speech impairment & $8(12 \cdot 7)$ & $2(3 \cdot 2)$ & - & $17(25 \cdot 8)$ & $8(12 \cdot 1)$ & - \\
\hline Cognitive disturbance & $5(7 \cdot 9)$ & - & & $8(12 \cdot 1)$ & $2(3 \cdot 0)$ & \\
\hline Personality change & $2(3 \cdot 2)$ & $1(1 \cdot 6)$ & & $6(9 \cdot 1)$ & $1(1.5)$ & \\
\hline Anxiety & $1(1 \cdot 6)$ & - & & $3(4 \cdot 5)$ & - & \\
\hline Sleeping problems & $4(6 \cdot 3)$ & - & & $6(9 \cdot 1)$ & - & \\
\hline Incontinence & $2(3 \cdot 2)$ & - & & $1(1.5)$ & - & \\
\hline Hearing impariment & $3(4 \cdot 8)$ & - & & $1(1.5)$ & - & \\
\hline Headache & $13(20 \cdot 6)$ & - & & $14(21 \cdot 2)$ & & \\
\hline
\end{tabular}

